\title{
Les enquêtes ethnobotaniques de Germaine Dieterlen (1903-1999)
}

Nouveau regard sur une ethnologue

Ethnobotanical inquiries by Germaine Dieterlen (1903-1999): a new look at an ethnologist

\section{Dominique Juhé-Beaulaton}

\section{(2penEdition}

\section{Journals}

Édition électronique

URL : http://journals.openedition.org/ethnoecologie/5989

DOI : $10.4000 /$ ethnoecologie.5989

ISSN : 2267-2419

\section{Éditeur}

Laboratoire Eco-anthropologie et Ethnobiologie

Référence électronique

Dominique Juhé-Beaulaton, « Les enquêtes ethnobotaniques de Germaine Dieterlen (1903-1999) », Revue d'ethnoécologie [En ligne], 17 | 2020, mis en ligne le 30 juin 2020, consulté le 10 décembre 2020. URL : http://journals.openedition.org/ethnoecologie/5989; DOI : https://doi.org/10.4000/ ethnoecologie.5989

Ce document a été généré automatiquement le 10 décembre 2020.

\section{$\Theta \Theta \Theta \Theta$}

Revue d'ethnoécologie est mis à disposition selon les termes de la licence Creative Commons Attribution - Pas d'Utilisation Commerciale - Pas de Modification 4.0 International. 


\title{
Les enquêtes ethnobotaniques de Germaine Dieterlen (1903-1999)
}

\author{
Nouveau regard sur une ethnologue \\ Ethnobotanical inquiries by Germaine Dieterlen (1903-1999): a new look at an \\ ethnologist
}

Dominique Juhé-Beaulaton

1 Le Muséum national d'Histoire naturelle de Paris conserve les archives et des herbiers de Germaine Dieterlen qui révèlent des aspects largement méconnus des intérêts et pratiques de terrain de cette ethnologue ${ }^{1}$ reconnue internationalement pour ses études sur les représentations du monde et les systèmes de pensée dogon et bambara au Mali (Piault 2001, Heusch 2001, Jolly 2016a). Formée à l'Institut d'ethnologie à Paris où elle a suivi les cours de Marcel Mauss (1870-1950), elle effectue ses premières enquêtes ethnographiques en Afrique en 1937 en partant en mission au Mali avec Solange de Ganay (1902-2003). Le lien recréé entre les archives et les herbiers de G. Dieterlen, quelque peu fastidieux compte tenu de la dispersion dans des fonds différents et en l'absence d'un inventaire précis, a néanmoins permis de retracer la démarche ethnobotanique que cette ethnologue a tenté de suivre à plusieurs reprises au cours de sa carrière. Ces documents ${ }^{2}$, archives et herbiers, révèlent l'application de ses recherches dans d'autres domaines que celui de l'ethnologie comme la pharmacologie, la muséologie ou le développement économique. Cet article a ainsi pour objet de présenter le recours à l'herbier comme un des instruments de travail de G. Dieterlen sur le terrain, faisant apparaître le rôle des intermédiaires et assistants de recherche locaux dans la mise en pratique de cet outil de recherche. Il montre également les ambiguïtés et les limites de la collaboration entre ethnologues et botanistes, comme le prouve la difficulté pour ces derniers à exploiter les collectes et les travaux de Dieterlen. Ceux-ci s'inscrivent dans le contexte de l'institutionnalisation de l'ethnobotanique en France au début des années 1960 autour de la personnalité de l'agronome botaniste Roland Portères (1906-1974), directeur du laboratoire d'ethnobotanique au Muséum, dans l'héritage de son prédécesseur, Auguste Chevalier (1873-1956) . 
Figure 1 : Portrait de Germaine Dieterlen, 1937 « Sur le bateau avec Geneviève Dieterlen »

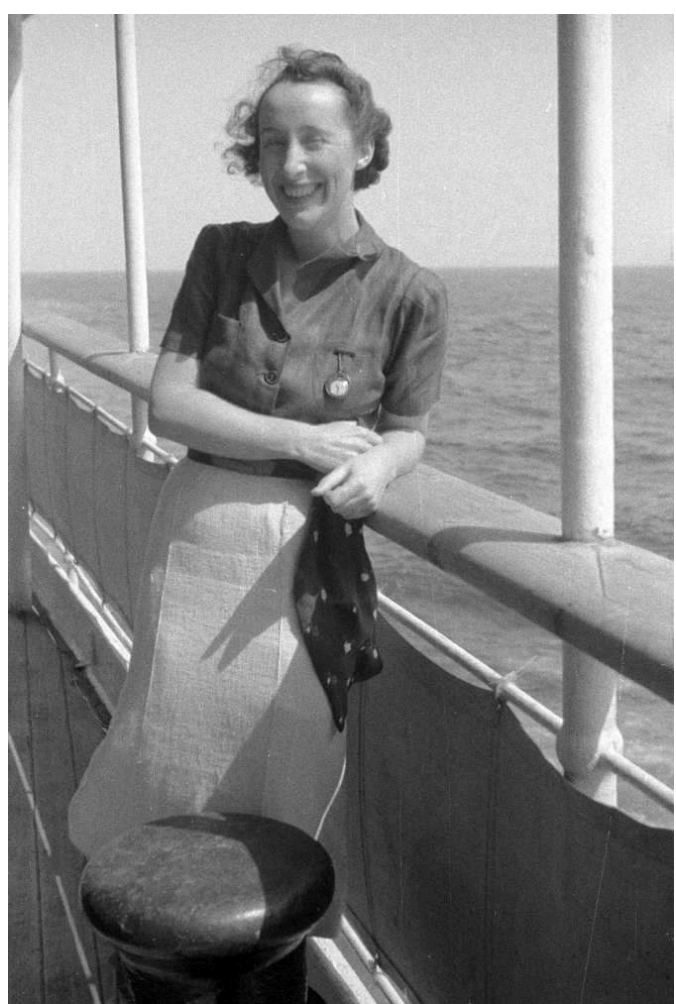

(c) Bibliothèque Éric-de-Dampierre, LESC, MAE, Université Paris Nanterre. Fonds Solange-de-Ganay. Cote : sdg_F_c_01_03

Lors de la célèbre "Mission ethnographique et linguistique » Dakar-Djibouti (1931-1933)4 dirigée par Marcel Griaule (1898-1956) à travers le continent africain, les membres de l'expédition sont chargés de collecter non seulement des objets ethnographiques mais aussi des spécimens naturels. Ils appliquaient ainsi les préceptes de Paul Rivet ${ }^{5}$, professeur d'anthropologie au MNHN, et les «Instructions d'ethnographie descriptive" de Marcel Mauss ${ }^{6}$. Ce dernier préconise de ne pas se limiter à la seule étude de la culture matérielle mais de la mener de front avec une enquête sur l'ethnobotanique et l'ethnozoologie. Ces conseils, repris par M. Griaule dans son propre cours et les «instructions sommaires pour les collecteurs d'objets ethnographiques » (Griaule \& Leiris 1931), seront suivis par de nombreux ethnologues ${ }^{7}$. Pour M. Griaule, « un herbier ou une collection de petits mammifères, si on les rattache à des usages humains, constituent des documents de même ordre qu'une collection d'objets fabriqués ou qu'un acte notarié » (Griaule $1957: 44)$.

Si les objets ethnographiques rapportés de ces expéditions l'emportent largement en nombre sur les spécimens naturels, Griaule et certains membres de son équipe ont cependant collecté " pour le muséum » selon l'expression couramment employée pour les apports des collaborateurs extérieurs à l'institution. Ainsi, le registre des entrées à l'Herbier du Muséum national d'Histoire naturelle de Paris mentionne le versement par M. Griaule d'un herbier de 250 échantillons le 7 mars $1933^{8}$. Deux autres ethnologues, membres des missions Griaule, ont également déposé leur herbier au MNHN : en 1938, Jean-Paul Lebeuf (1907-1994) verse 75 échantillons provenant du Cameroun (quatrième mission Sahara-Cameroun, 1936-1937) et Solange de Ganay, 250 échantillons récoltés en 
1937 à Sanga au Mali'.''assemblée des Professeurs du Muséum de décembre 1936 avait en effet accordé une subvention de $2000 \mathrm{Fr}$ à S. de Ganay et G. Dieterlen, chargées d'une mission d'ethnologie en Afrique occidentale (A.GD carton 20). Au cours de cette mission de sept mois en pays dogon, S. de Ganay fait des enquêtes en ethnozoologie et ethnobotanique dans la région de Sanga ${ }^{10}$ et elle poursuit le relevé du plan parcellaire du village des Ogol commencé en 1935 lors de la mission Sahara-Soudan (Lebrun 1937). Pour ce premier terrain en binôme ${ }^{11}$, Germaine Dieterlen n'est restée que deux mois et s'est concentrée sur les cultes dogon, sujet de son mémoire de fin d'études de l'École pratique des hautes études (sciences religieuses) en 1940. Elle sera ensuite de toutes les « missions Griaule » et s'attachera alors à recueillir des échantillons d'herbier.

\section{L'IFAN et les premières enquêtes ethnobotaniques (1950-1953)}

G. Dieterlen réalise ses premières collectes ethnobotaniques en pays dogon, àSanga dans les falaises de Bandiagara en 1950 et 1951 à la demande de Théodore Monod (1902-2000), directeur de l'Institut français d'Afrique noire (IFAN) basé à Dakar. Les trois cents spécimens récoltés ont été déposés et déterminés à l'IFAN (A.GD 10). Elle réalise également pour le préhistorien Guy de Beauchesne du Musée de l'Homme une enquête chez les Dogon sur l'utilisation de plantes ichtyotoxiques pour la pêche à partir d'un questionnaire que celui-ci lui a très probablement remis, véritable fiche ethnobotanique (Figure 2). Il y est précisé de porter attention au nom vernaculaire de la plante, son lieu de collecte, son habitat (savane, forêt...), la nature du sol, ses modes de préparation...et de récolter des échantillons aussi complets que possible (différentes parties: fleur, feuille, racines...). G. Dieterlen publie ses résultats sur les plantes ichtyotoxiques dans Notes africaines en section botanique en 1952, en se basant sur ses travaux antérieurs pour les aspects religieux et en précisant les noms scientifiques des plantes. Elle indique en note qu'un échantillon de "polo » était insuffisant pour être précisément déterminé : seul le genre Sanseviera a pu être spécifié ${ }^{12}$. G. Dieterlen prend $l^{1}$ habitude de donner les noms scientifiques des plantes dans ses publications ${ }^{13}$. 
Figure 2 : Questionnaire d'enquête sur les plantes ichtyotoxiques pour la pêche

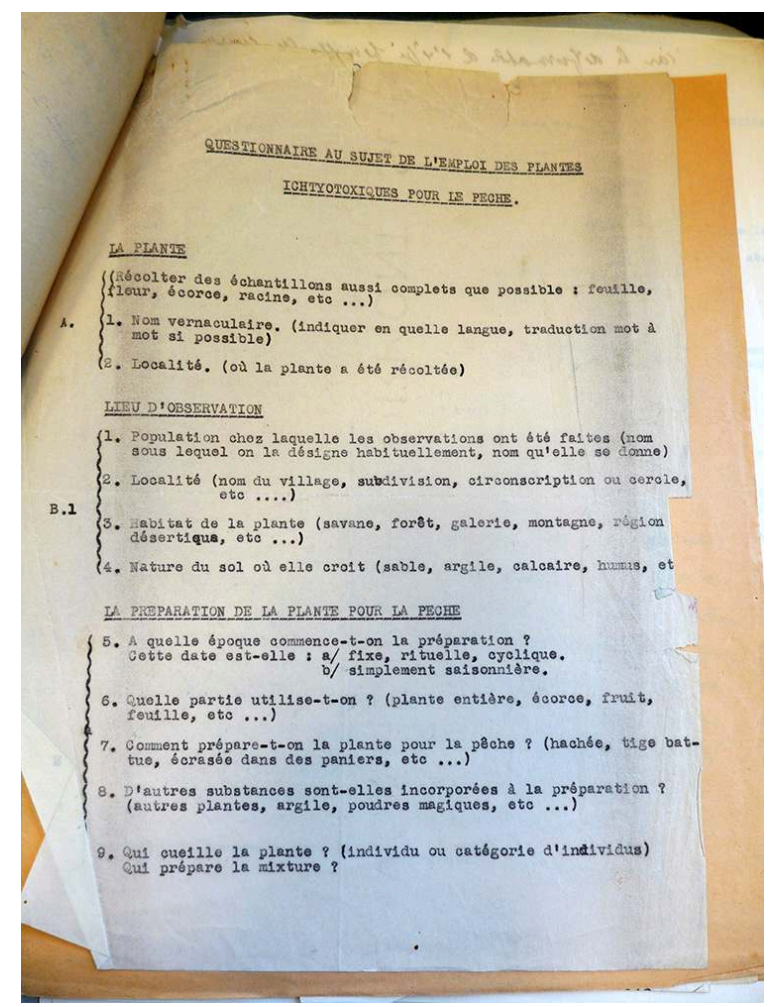

Archives G. Dieterlen, carton 10. MNHN Paris. Photographie D. Juhé-Beaulaton

5 C'est également à partir de cet herbier ${ }^{14}$ que G. Dieterlen a rédigé « la classification des végétaux chez les Dogon ", article publié dans le Journal de la Société des Africanistes en $1952^{15}$. Dans son introduction, elle précise que l'herbier a servi de point de départ aux enquêtes. Elle ne développe pas dans ce texte ses méthodes d'enquêtes mais donne seulement son interprétation des propos de ses informateurs cités en note :

"Ce classement a été établi à Sanga avec les principaux informateurs suivants: Ongnonlou, patriarche de Go; Akoundyo de BArA, patriarche de Sodamma, guérisseur ; AmbaraDolo, d'Am-taba ; Apourali et Koguema Dolo, de Doziou Orey ; Yébéné de BArA, prêtre du Totem Yébéné de Sodamma; ainsi qu'avec Manda, prêtre du Binou Manda d'Orosongo. Les étymologies fournies ici sont indigènes. Encore que ce ne soit pas toujours expliqué, elles reflètent des associations d'idées relatives au système de classification des plantes ».

Parmi les personnes rencontrées figurent - sans surprise - les informateurs privilégiés de M. Griaule dont Ambara Dolo et Koguema Dolo, principaux collaborateurs, guides et interprètes des missions ethnographiques en pays dogon (Figure 3). Non seulement ils traduisaient, mais ils synthétisaient, complétaient, commentaient, les discours des autres informateurs dogon et collectaient spécimens naturels et objets ${ }^{16}$. Cet essai de classification propose une catégorisation des végétaux en vingt-quatre familles chacune étant en relation avec une partie du corps humain. L'ensemble forme un système de correspondances «cosmo-biologiques" représentant la "somme des connaissances dogon» (p. 115). Les plantes de l'herbier sont classées en fonction de ces familles et nommées selon certaines plantes éponymes qui occupent une place particulière dans la cosmogonie ou la société dogon. "Les plantes récoltées et déterminées qui font partie [d'une] famille sont énumérées ensuite par ordre alphabétique, ordre qui n'est évidemment pas celui des Dogon » (p. 124) : par exemple 
le fonio (Digitaria exilis), associé « au point osseux de la clavicule droite ", à l'âme et à la procréation, représente la première famille par son rôle dans la création du monde (p. 125). Dans la dixième famille associée à la respiration et aux mouvements du corps, elle reprend les plantes citées dans son article sur les plantes ichtyotoxiques avec comme plante éponyme polo (Sanseviera sp.) (p. 140).

Figure 3 : Portrait d'AmbaraDolo

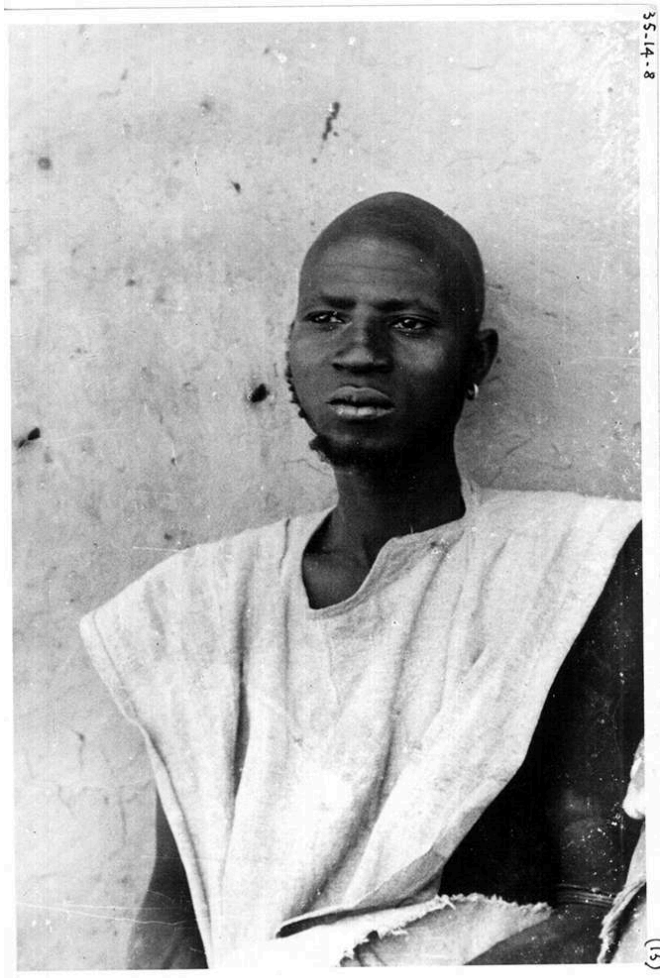

(CM. Griaule, Marcel Fonds Marcel-Griaule. Mission Sahara-Soudan (1935) Bibliothèque Éric-deDampierre, LESC, MAE, Université Paris Nanterre (Cote : fmg_E_a_03_L_14_008)

7 Sans reprendre toutes les catégories définies, il ressort de cette classification une liste de plantes ordonnées selon leur utilisation associée à une partie du corps humain et à la cosmogonie : les plantes purificatrices ; les plantes tinctoriales et à tannins utilisées par le cordonnier; les plantes liées à la chasse et à la guerre; les plantes alimentaires sauvages et cultivées en agriculture, pour ne citer que quelques exemples. Ces différentes catégories sont d'ailleurs poreuses car certaines plantes, classées dans les "plantes d'eau», peuvent aussi être utilisées pour purifier. Certaines espèces représentent à elles seules une "famille", comme le Prosopis africana en raison de son « rôle mythique " particulier, associé au forgeron. Cet inventaire des plantes connues des Dogon s'appuie sur les déterminations botaniques qui - elle le reconnaît - sont incomplètes dans ces premiers travaux. Ainsi pour les acacias, elle mentionne différentes "variétés " dont les identifications étaient alors en cours et nombre de plantes ne sont pas déterminées botaniquement. Cette classification ressemble plus à une ébauche et un point de départ pour de nouvelles recherches; G. Dieterlen écrit d'ailleurs qu'il s'agit d'un "premier article» appelant une suite et elle invite les chercheurs à s'intéresser aux systèmes de classification "indigènes ", à l'instar d'A. Chevalier ${ }^{17}$ (1937) dont elle cite un article sur « les plantes magiques cultivées par 
les Noirs d'Afrique». Celui-ci dresse un inventaire des plantes souvent toxiques utilisées à la fois en médecine, religion et magie. Cette relation a retenu l'attention de G. Dieterlen qui a basé sa classification sur le système religieux dogon. Ceci sera critiqué par un certain nombre d'ethnologues, dont C. Friedberg (1974), connue pour ses recherches en ethnoscience. Selon cette auteure, certains travaux sur les systèmes de classification indigène traduisent davantage les théories de leurs "inventeurs", basées notamment sur les cosmogonies pour Griaule et Dieterlen et non sur les taxinomies locales ${ }^{18}$.

Pour G. Dieterlen, la «catégorie végétale » a un "rôle de représentation générale de l'univers » mais elle constitue aussi « la base de l'alimentation et de la pharmacopée ». Si cette classification est connue de la plupart des adultes cultivateurs, elle est « l'objet d'une véritable science de la part des guérisseurs» (p.116). Elle n'aura de cesse de compléter son inventaire des plantes en l'élargissant aux plantes médicinales. G. Dieterlen poursuit ainsi ces enquêtes ethnobotaniques lors de ses nombreuses missions dans plusieurs pays africains, mais le plus souvent en pays dogon au Mali où elle a effectué de très nombreux séjours ${ }^{19}$. En 1953, elle recueille à nouveau un herbier de 165 plantes dont les noms vernaculaires dogon figurent dans son carnet de terrain de 1953 avec des numéros de collecte. Son écriture appliquée semble indiquer que cette liste a été établie après la collecte, peut-être à partir des échantillons ou bien recopiée (p. 453 à 457). L'écriture des pages suivantes, plus difficilement lisible, montre une prise de notes rapide pendant l'entretien sur les usages des plantes ${ }^{20}$. Son objectif était de réviser la classification des végétaux précédemment publiée, témoignant d'une volonté constante de faire évoluer celle-ci en fonction des nouveaux développements de ses informateurs.

9 L'enquête ethnobotanique semble se dérouler en trois temps ${ }^{21}$ :

- la collecte d'échantillons ;

- l'établissement de leur liste soigneusement transcrite phonétiquement et numérotée ;

- l'entretien sur les usages basé sur les échantillons, support matériel de la mémoire qui active ou libère la parole.

Elle précise dans son rapport de mission de 1953 que ses enquêtes orales ont porté sur la «structure sociale des Dogon en relation avec leur système de représentation du monde [...], la caste des forgerons, sur la médecine (en rapport avec l'herbier et la classification des végétaux) ». Cet herbier est également déposé à l'IFAN, alors que des empreintes palmaires et des graines ont été remises au Musée de l'Homme (Département Anthropologie) ${ }^{22}$.

11 En 1954, T. Monod envoie de l'IFAN à Marcel Griaule la liste des noms de plantes récoltées par G. Dieterlen en précisant: «il y a plusieurs blancs mais certains échantillons étaient malheureusement très insuffisants $»^{23}$. La qualité des échantillons sera effectivement souvent trop médiocre pour permettre leur identification.

\section{Dieterlen et l'ethnopharmacologie}

12 Tout au long de sa carrière, G. Dieterlen a montré beaucoup d'intérêt pour les plantes médicinales, à Sanga au Mali comme sur les lieux de ses autres terrains d'enquête. Dans son article sur la classification des végétaux chez les Dogon en 1952, elle pressent que 
ces recherches peuvent dépasser le cadre de l'ethnologie et intéresser d'autres sciences

\begin{abstract}
« Devant l'intérêt évident que présente, peut-être pour la médecine et la pharmacie elles-mêmes, en tous les cas pour leur histoire, cette connaissance, nous souhaitons apporter - et que d'autres apportent avec nous - le plus grand nombre de matériaux possibles en constituant en Afrique des herbiers classés selon les méthodes indigènes et en recueillant tous les usages de leurs éléments. Ces matériaux livrés aux chercheurs appartenant à d'autres disciplines permettront des analyses et peut-être des expériences. » (Dieterlen 1952b : 156-157).
\end{abstract}

Ce passage de son article annonce les collaborations à venir avec des pharmacologues français. Elle a d'ailleurs pris contact avec le laboratoire de pharmacologie et de matière médicale de la Faculté de médecine de Paris, comme l'atteste cette lettre datée du 12 octobre 1953 que lui adresse le professeur René Hasard (1886-1974) :

«Madame,

Je m'excuse d'avoir tant tardé à vous donner une réponse positive aux questions que vous m'avez posées touchant les possibilités d'études pharmacologiques des plantes africaines.

De l'examen du dossier que vous avez bien voulu me confier, il me semble ressortir qu'il serait intéressant de commencer par les végétaux suivants dont la liste est ajoutée à ma lettre. Leur étude représente déjà pour nous une source de travail important et que nous entreprendrons volontiers. Nous pourrions ensuite passer à l'étude d'autres végétaux.

Je vous remercie de bien vouloir nous offrir des possibilités de recherche dont les pharmacologistes sont toujours friands.

signé R. Hazard $»^{24}$.

Quelle suite a été donnée à cette ébauche de collaboration ? Les archives Dieterlen n'en disent rien et la liste mentionnée par R. Hasard ne s'y trouve pas ${ }^{25}$. Cependant, au cours des années 1950, la recherche scientifique sur les plantes médicinales de la zone intertropicale s'organise en partenariat avec la Faculté de pharmacie de Paris, l'office de la recherche scientifique et technique d'Outre-mer (ORSTOM) et le Centre d'études et d'informations ethnobotaniques créé en 1957 sous l'impulsion de R. Portères au sein du laboratoire d'Agronomie tropicale (Portères 1957). Ceci sous-entend le développement de relations avec les ethnologues ${ }^{26}$. C'est d'ailleurs au MNHN que G. Dieterlen va désormais déposer ses échantillons de plantes, quelques-uns à l'Herbier général et la majorité au laboratoire dirigé par Portères (A.GD carton 20). La collaboration d'ethnologues et de botanistes agronomes de ce laboratoire, déjà initiée par son premier directeur, Auguste Chevalier, se poursuivra de longues années ${ }^{27}$.

\title{
L'enquête sur les plantes médicinales en Côte d'Ivoire (1959-1966)
}

Profitant d'un séjour en Côte d'Ivoire en décembre 1959 à l'occasion d'un colloque sur "La recherche scientifique et technique " à Abidjan, G. Dieterlen entreprend une enquête orientée spécifiquement sur les plantes médicinales dans un village à une trentaine de kilomètres à l'est d'Abidjan ${ }^{28}$. Elle se rend à Bregbo, village du sud-est ivoirien, où exerce le prophète harriste Albert Atcho (1903-1980) ${ }^{29}$. Elle a ainsi l'opportunité d'observer la thérapeutique appliquée à ses malades et recueille alors "des plantes pharmaceutiques en vue de leurs études par le laboratoire d'Ethnobotanique du Muséum d'histoire naturelle et enquête sur leurs usages $»^{30}$.Ce passage à 
Abidjan correspond aux dates des enquêtes sur les plantes médicinales des pharmaciens Armand Bouquet et Maurice Debray qu'elle a peut-être rencontrés lors de ce colloque ${ }^{31}$. Aucun document d'archive ne permet à ce jour d'attester de relation entre l'ethnologue et ces pharmaciens. Jean Rouch (1975:20) nous apprend cependant que Bouquet est le premier à identifier des plantes utilisées par Albert Atcho à Bregbo, auprès de qui il a aussi recueilli des informations ${ }^{32}$. La première série de plantes recueillies à Bregbo par G. Dieterlen, introuvable au Muséum, est peut-être restée au Centre ORSTOM d'Adiopodoumé en Côte d'Ivoire où étaient basés A. Bouquet et M. Debray à la fin des années $1950^{33}$.

Figure 4 : Portrait d'Atcho par M. Debray

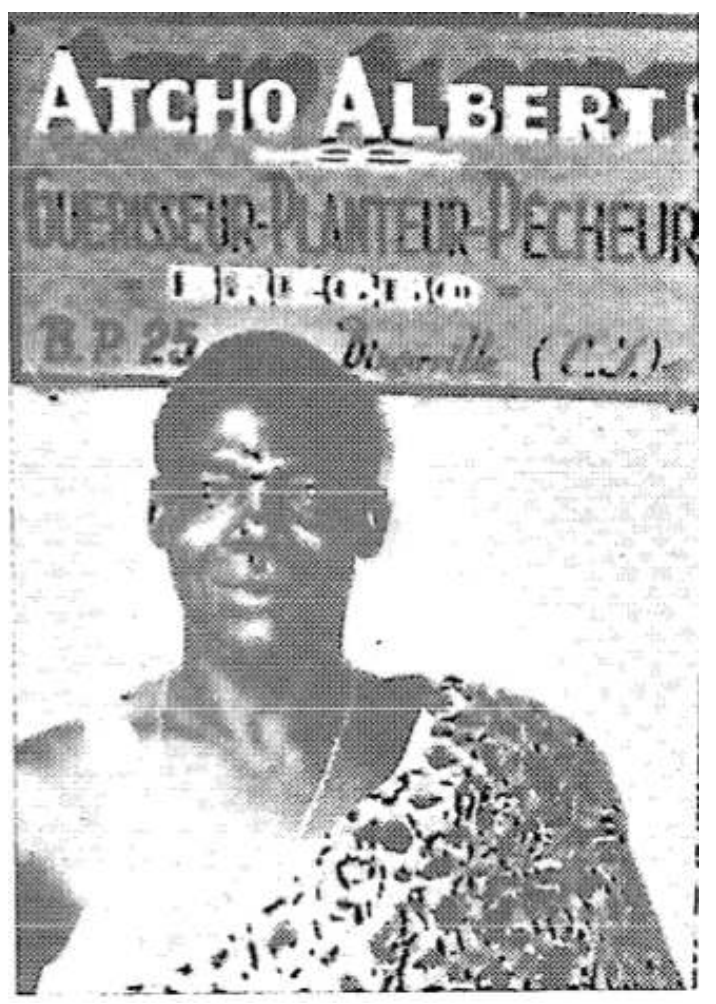

Cliché ORSTOM, in Bouquet et Debray (1974 : 177, planche photographique $n^{\circ} 1$.

En 1962, G. Dieterlen poursuit ce travail sur les plantes médicinales lors d'une étape à Abidjan après le premier congrès international des Africanistes à Accra et avant de repartir par la route à Niamey avec Jean Rouch (1917-2004) $)^{34}$. Elle se rend avec celui-ci à Bregbo et reprend ses observations des soins prodigués aux malades par le prophète Atcho et ses adjoints. Cette thérapeutique qui, selon ses mots, "se veut globale » est basée à la fois sur des confessions, des rituels et des soins. J. Rouch (1963) finalise le tournage d'un film et G. Dieterlen assiste à diverses cérémonies organisées par A. Atcho pour la communauté de malades groupés autour de lui. Elle remarque que nombre d'arbres et de végétaux étaient plantés à Bregbo à des fins thérapeutiques et religieuses (Rouch 1975 : 20). Elle relate dans son rapport de mission de 1962 :

« la mise au point d'un herbier des plantes médicinales utilisées par Monsieur Atcho pour soigner les malades qui viennent le consulter [...]. Les plantes recueillies seront examinées et déterminées par les laboratoires du Muséum d'histoire naturelle. Tous les renseignements recueillis par nos informateurs sur l'usage médical de ces plantes feront l'objet de publications $»^{35}$. 
Il s'ensuit une vaste enquête collective pluridisciplinaire sur la communauté de Bregbo subventionnée par le CNRS, pilotée par Jean Rouch et Colette Piault de 1962 à $1966^{36}$. Les confessions des malades soignés par Atcho et son personnel, recueillies et transcrites par les enquêteurs, sont envoyées à Paris pour être analysées par le groupe de chercheurs africanistes de l'EPHE ${ }^{37}$. L'enquête ethnobotanique sur les usages thérapeutiques des plantes, initiée en 1959, a bien été menée. Un des objectifs était de réaliser une pharmacopée qui, en principe, devait être publiée dans la publication collective prévue des résultats de l'équipe. G. Dieterlen, à l'origine de cette approche, était initialement chargée de la rédaction de cette partie. L'extrait de son rapport cité précédemment mentionne le rôle des "informateurs locaux" dans le recueil des données et des herbiers. La récolte des échantillons d'herbier n'a, en fait, pas été réalisée par Germaine Dieterlen, mais par Opokou Jean Vangah et Dayé Nanguy Gabriel, assistants du prophète Albert Atcho ${ }^{38}$. Agissant sur sa commande dans le cadre du projet CNRS, ils étaient rétribués pour ce travail qui ne se limitait pas à l'ethnobotanique mais aussi à la transcription des confessions des malades. Ils envoyaient les échantillons de plantes à G. Dieterlen qui les faisaient ensuite déterminer au Muséum soit par Roland Portères, soit par Hubert Gillet ${ }^{39}$. Ils sont tous deux mentionnés dans plusieurs documents des cartons 3 et 9 des archives et sur les échantillons eux-mêmes. Ces derniers, souvent de qualité insuffisante pour permettre leur identification, sont accompagnés de fiches bleues présentant les noms des plantes en langue vernaculaire (agni, attié, ebrié, alladian, mbatto, abouré), les usages pharmaceutiques, leur numéro de collecte commençant par « $2^{\text {ème }}$ série $n^{\circ} 1$ à 843 » et parfois la date, entre octobre/novembre 1965 et janvier/avril 1966. Les numéros reportés sur les fiches donnent le nombre d'échantillons de l'herbier, soit un total de 843 parts dont une partie seulement a été conservée ${ }^{40}$. 
Figure 5 : Échantillon d'Hibiscus non monté, $n^{\circ} 100,2^{\text {ème }}$ série Bregbo, Côte d'Ivoire. Partiellement identifié. Indication : "contre un bubon qui commence"

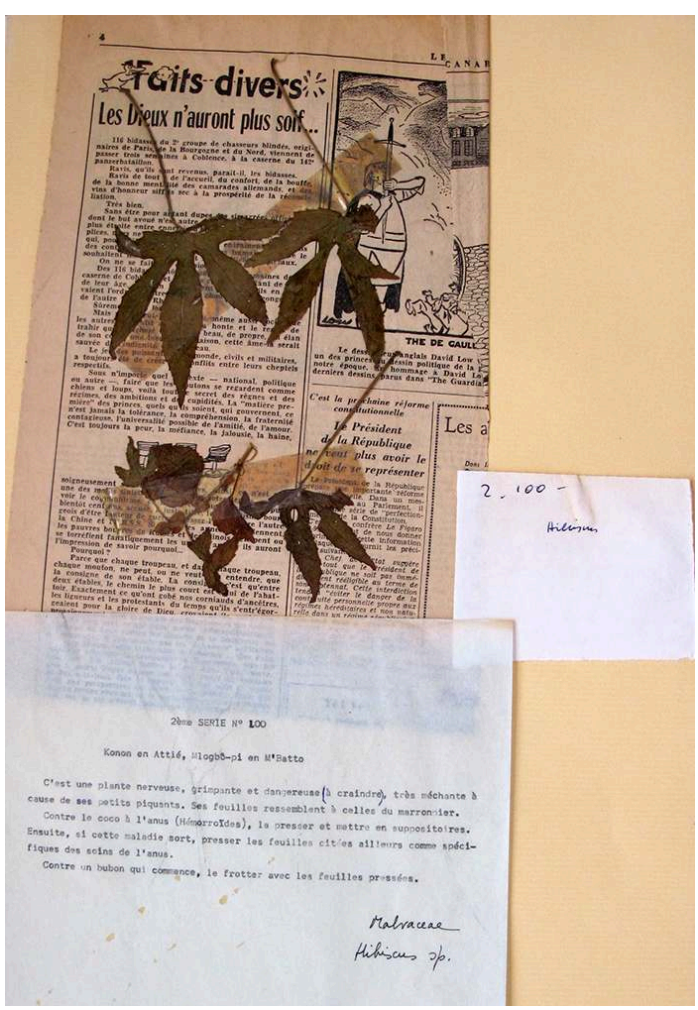

Photographie S. Juraver informateurs locaux dans un certain embarras financier. Ils expriment leur inquiétude dans plusieurs lettres adressées à G. Dieterlen la même année, lui signalant la poursuite des envois de plantes et de mémoires et leur attente en échange d'une rétribution ${ }^{41}$.

G. Dieterlen a toujours reconnu leur travail qu'elle évoque dans le brouillon de l'avantpropos qu'elle a commencé à rédiger en vue de la publication des résultats ${ }^{42}$ :

«[il] nous est apparu immédiatement le rôle joué par l'application quotidienne de techniques relevant de la médecine traditionnelle en Afrique noire, à savoir l'usage de plantes médicinales.

Nous avons donc, avec l'accord d'Albert Atcho, demandé à ses deux adjoints, Gabriel Dayé Nangwi et Jean Vangah, de constituer un herbier en collectant ces végétaux, ceci aux fins d'identification par des laboratoires, ces identifications constituent le premier acte d'une étude systématique complète de la thérapeutique. L'herbier, comprenant 850 échantillons, a été confié dans ce but au laboratoire d'ethnobotanique du professeur R. Portères.

La présentation de la pharmacopée et de la thérapeutique traditionnelle utilisées à Bregbo a été réalisée sous forme de tableaux déterminés par la nomenclature des troubles et symptômes maladifs auxquels s'appliquent les échantillons de l'herbier. Cette nomenclature présente l'avantage de proposer une approche des connaissances anatomiques, physiologiques, psycho-physiologiques des populations intéressées, ainsi que la terminologie des symptômes et les affections. Elle a également pour but de souligner l'usage médical des plantes recueillies, dont la classification végétale indigène, qui englobe certainement d'autres catégories que celles des affections (alimentation, techniques, rituel etc....), ne nous est pas connue. Une telle classification existe dans d'autres ethnies d'Afrique noire (au Mali : Dogon et Bambara ; au Dahomey)». 
20 présente comme une ébauche de recherches à venir. Elle indique l'absence d'information sur les guérisseurs (leur formation, leur rôle social, les rituels...), sur les conditions de recueil des échantillons de plantes (époque de l'année, orientation dans l'espace...), sur les éléments autres que végétaux utilisés en associations et elle alerte les chercheurs sur l'urgence et l'importance d'une telle approche redoutant une perte de ces savoirs.

Le projet de publication des résultats de cette vaste enquête collective débute en 1966. Une partie sur la pharmacopée était initialement prévue mais, dès 1967, lors d'une réunion éditoriale de l'ouvrage sur Bregbo à laquelle G. Dieterlen, absente, était représentée par Aline Attal, il est décidé que le travail sur les plantes médicinales, d'un volume trop important - près de 850 échantillons et fiches -, fera finalement l'objet d'une publication autonome. Seules « une dizaine de pages cependant seraient publiées à titre d'exemple dans l'ouvrage général » annoncées dans le plan provisoire de l'ouvrage dans la troisième partie consacrée à la thérapeutique sous le titre «III.2. Pharmacopée ». En 1968, le plan prévoyait 20 pages attribuées à G. Dieterlen et A. Attal. Des textes de présentation de la pharmacopée accompagnés de tableaux et d'exemples de plantes identifiées botaniquement ont bien été remis. Des modifications ont été demandées et il semble que la version finale, réclamée par C. Piault à G. Dieterlen pour février 1969 n'a jamais été rendue. L'ouvrage, finalement publié en 1975 par Marc Augé, René Bureau et Colette Piault sous le titre Prophétisme et thérapeutique. Albert Atcho et la communauté de Bregbo, ne comporte pas de présentation de la pharmacopée. La contribution de G. Dieterlen, fondée sur un inventaire de plantes et le recueil de données empiriques, s'éloignait de plus en plus des normes éditoriales en ethnologie (Jolly 2001 : 181), ce qui peut justifier, peut-être, son absence dans le livre sur Atcho. La publication indépendante de la pharmacopée n'a, elle non plus, jamais été réalisée ${ }^{43}$. Colette Piault, dans une lettre à Germaine Dieterlen datée de 1976, lui annonce qu'il a fallu faire des coupes pour la publication et elle espère qu'elle n'a pas été trop déçue du résultat. Elle exprime à la fin de sa lettre que la reprise des analyses des plantes annoncée par J. Rouch est une bonne nouvelle (A.GD 3 : dossier « Herbier Bregbo »), ce qui sous-entend des problèmes posés par l'identification de nombre d'échantillons pouvant expliquer l'inaboutissement de ce travail.

Les résultats de l'enquête ethnobotanique n'ont certes pas été publiés, même séparément de l'étude sociologique ; ils sont restés présentés sous forme de tableaux et de fiches se référant à l'herbier conservés dans le dossier « médecine» du carton 3 des archives. Celui-ci comprend plusieurs types de documents :

- Les manuscrits originaux rédigés en français local par le secrétariat du prophète Atcho comportant des informations sur les usages ; (Figure 6)

- Un Index alphabétique des soins thérapeutiques administrés à partir des plantes de l'herbier de Bregbo » (exemples : application cutanée, bains, infusion, inhalation, lavement, ...) en indiquant les numéros de références des plantes de l'herbier que l'on retrouve dans les fiches et les tableaux (fascicule dactylographié);

- Une nomenclature des maladies auxquelles s'applique la pharmacopée de Bregbo: accouchement, blennorragie, système digestif (constipation, diarrhée, digestion, dysenterie, foie...), voies respiratoires (toux, rhume...), peau (dartre, furoncle...), organes génitaux, système nerveux (troubles mentaux, folie...), maladie du sommeil, paludisme, rougeole, symptômes liés à l'envoûtement et à la magie, varicelle, variole... 
(Figure 7 : Index des maladies à Bregbo) ;

- Des tableaux présentant les utilisations des plantes classées par type de maladie et la thérapeutique associée en faisant référence aux plantes, à leur nom vernaculaire et parfois leur identification botanique. (Figure 8 : exemple Scoparia dulcis $n^{\circ} 250$ ) ;

- Des fiches bleues dactylographiées associées aux parts d'herbier $2^{\text {ème }}$ série $n^{\circ} 1$ à 414 : plantes et usages médicinaux avec les noms vernaculaires dans les différentes langues (ebrié, alladian, mbatto, abouré) dont un double se trouve dans les échantillons d'herbier correspondant. (Voir exemples dans Figures 4 et 8 )

Figure 6 : Manuscrit original rédigé en français local par le secrétariat du prophète Atcho comportant des informations sur les usages. Référence à l'échantillon $n^{\circ} 100$. A.GD 20

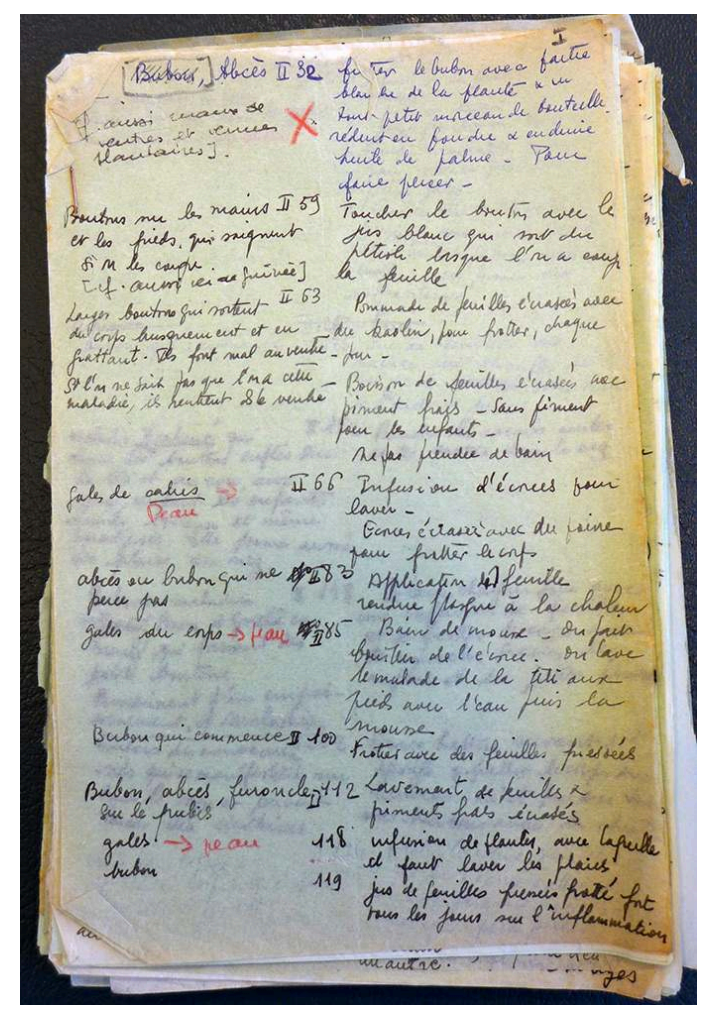

Photographie D. Juhé-Beaulaton 
Figure 7 : Nomenclature des maladies auxquelles s'applique la pharmacopée de Bregbo (A.GD 20)

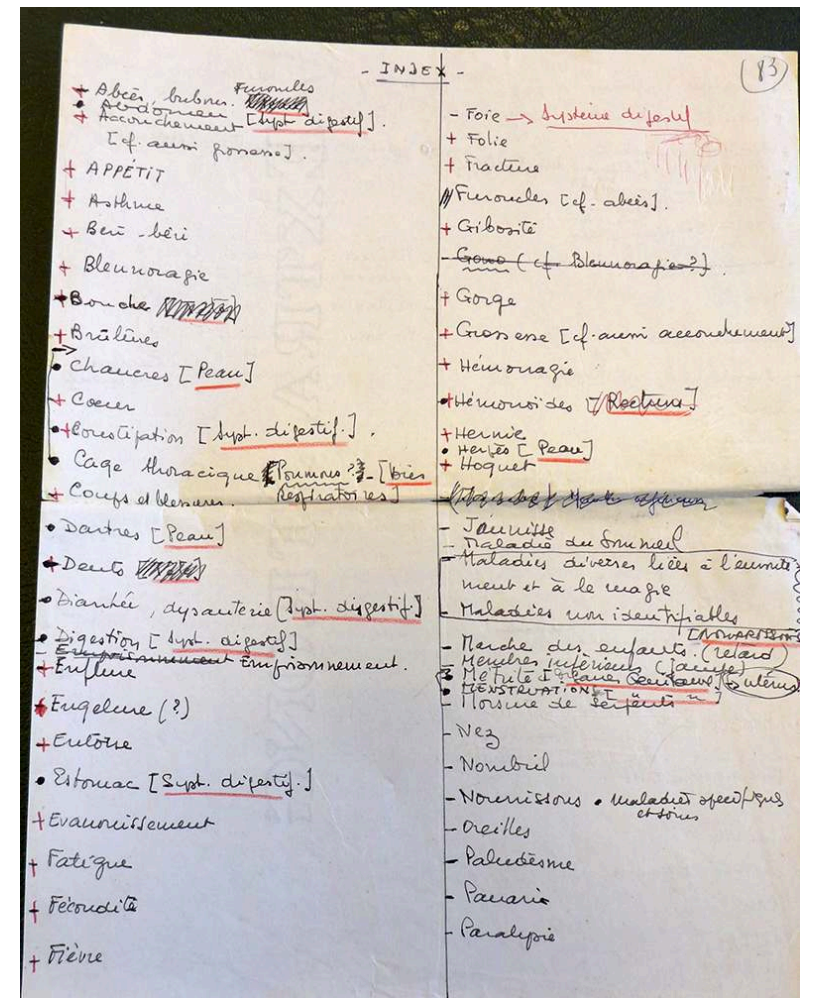

Photographie D. Juhé-Beaulaton

Figure 8 : Tableau présentant les utilisations des plantes classées par type de maladie et la thérapeutique associée en faisant référence aux plantes, à leur nom vernaculaire et parfois leur identification botanique

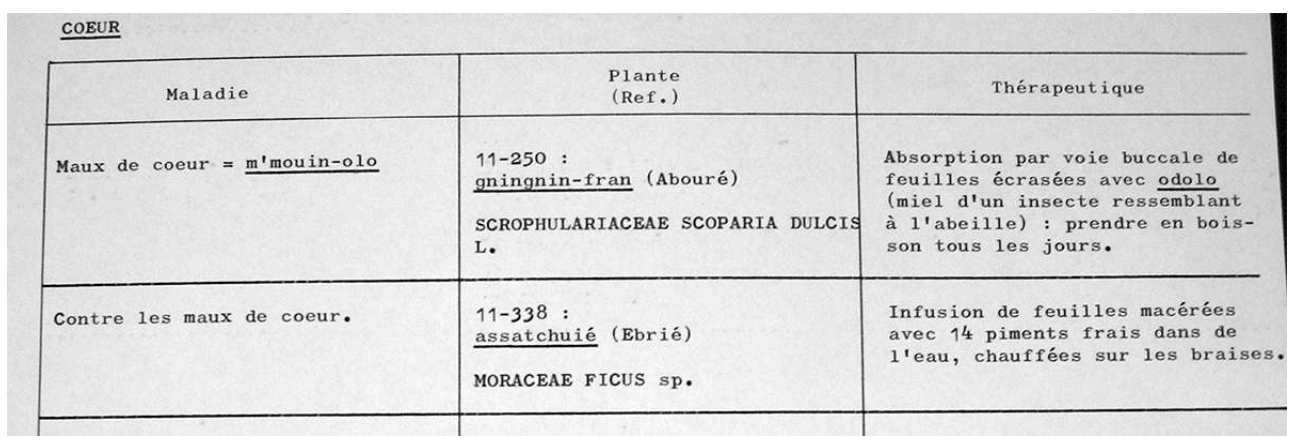

Photographie D. Juhé-Beaulaton

L'intérêt de G. Dieterlen pour les plantes médicinales allait au-delà de la collecte de plantes et des savoirs associés qui pouvait lui donner des informations sur «l'interprétation des connaissances physiologiques et des maladies» des personnes interrogées, connaissances reflétées dans la terminologie utilisée pour décrire les maladies et les symptômes ${ }^{44}$. En effet, une correspondance retrouvée dans ses archives mentionne des contacts avec un laboratoire pharmaceutique basé à Paris : un dénommé « Faral » ${ }^{45}$, auteur d'une lettre à l'en-tête du laboratoire M.J. Ravel datée du 22 juin 1966 et adressée à Roger $\mathrm{Heim}^{46}$, lui demande en premier lieu les coordonnées de G. Dieterlen. Puis Faral mentionne un "accord de principe » pour la création d'un « service du CNRS (Mme D.) »- Mme D. étant très vraisemblablement G. Dieterlen - au 
sein du laboratoire Ravel dans un nouveau bâtiment et attend pour ce faire une " demande officielle ». Il évoque ensuite une discussion qu'il a eue avec son ami Henri Laugier $^{47}$ au sujet d'un projet de création d'un institut d'études pharmacologiques. Enfin, Faral précise à Heim qu'il lui envoie une copie d'une lettre de Laugier dont le sujet est encore la création de cet institut demandant à Heim de remettre à Mme D. le contenu de cette lettre. Il conclut qu'elle « en tirera les conclusions elle-même ».

Henri Laugier (1888-1973), premier Directeur du CNRS, a joué un rôle important dans l'organisation de la recherche scientifique en France et dans la création d'un certain nombre d'institutions d'ampleur nationale et internationale dont certaines sont restées à l'état de projet. Sa lettre à Faral laisse entendre que ce dernier lui a demandé son avis et très probablement son appui pour la création de cet institut de recherches pharmacologiques ${ }^{48}$ :

«Par ailleurs je suis d'accord avec vous sur la pharmacologie [...] qui mériterait un mécanisme d'étude officiel beaucoup plus riche que ceux existant. J'ai souvent milité (sans résultat jusqu'ici) aux temps de l'«Empire» et de la Grande Colonisation pour que soit créé à Paris un «Institut d'étude pharmacologique largement doté avec mission de faire l'inventaire de toutes les ressources pharmacologiques que l'on peut trouver dans la flore, éventuellement la faune, de nos colonies; et d'étudier toutes les propriétés de ces produits ». - Je pense que même sans "Empire ", le problème peut être repris. - Les grandes et puissantes firmes pharmaceutiques s'honoreraient si elles se substituaient aux officiels d'État en pleine carence pour créer collectivement un de ces laboratoires d'une importance, je suis d'accord avec vous, certainement considérable. [...]

Il faudra que nous parlions de tout cela.

Très affectueusement, fidèlement et frat (abréviation suivie de trois points en triangle)

Signé Henri Laugier $»^{49}$.

Faral comptait certainement sur le réseau de Laugier et son entregent - il était francmaçon - pour appuyer ce projet dont G. Dieterlen était partie prenante, voire l'instigatrice auprès du laboratoire Ravel. Depuis ses premiers contacts avec la faculté de pharmacie de Paris en 1953, G. Dieterlen pensait la collecte ethnobotanique comme une étape précédant les analyses pharmacologiques, ce qui n'est pas sans rapport avec le développement des recherches sur les plantes médicinales, particulièrement en Côte d'Ivoire, au cours des années 1950 peu de temps avant les indépendances des pays colonisés. Ce projet est, semble-t-il, resté sans suite, mais G. Dieterlen n'a pas pour autant cesser de s'intéresser aux plantes médicinales. 
Figure 9 : Présentation de Scoparia dulcis récoltée à Bregbo

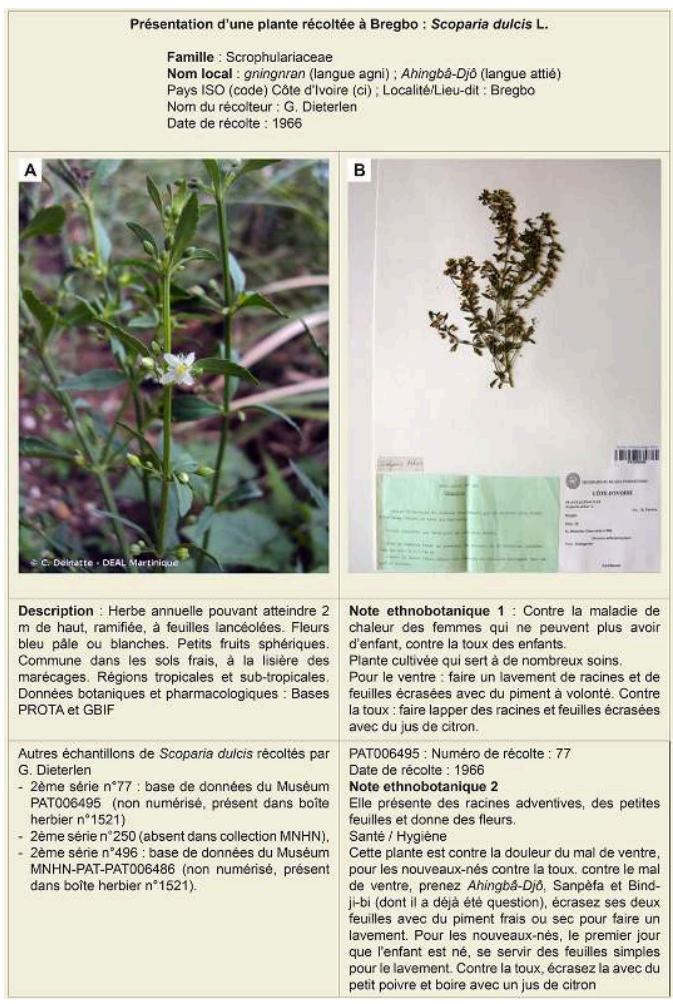

A : Scoparia dulcis @ C. Delnatte https://inpn.mnhn.fr/espece/cd_nom/447612

B : Part d'herbier de Scopularia dulcis L., «Pharmacopée Bregbo » accompagnée d'une fiche bleue présentant le nom vernaculaire de la plante, ses usages pharmaceutiques, son numéro et la date de collecte. Photographie S. Juraver

\section{Une ethnologue au service du laboratoire Debat (1976-1978)}

En pays dogon où elle a régulièrement effectué des missions, elle n'a cessé de collecter des plantes dans l'intention de reprendre sa classification de 1952 en la complétant de nombreuses plantes médicinales. Au cours des années 1970, à la fin de sa carrière, elle envoie encore au laboratoire d'ethnobotanique des échantillons pour identification comme l'atteste une lettre adressée à Roland Portères en 1973. Celle-ci témoigne de la permanence des relations de G.Dieterlen avec les membres de ce laboratoire (Figure 10) et de sa constance à collecter des plantes :

«Cher Monsieur Portères,

Ce mot est seulement pour vous remercier très chaleureusement de ce que vous avez bien voulu faire pour l'herbier Dogon. C'est infiniment précieux pour moi et nécessaire pour la publication. [...] Je vais me procurer les végétaux que vous avez signalé et dont l'identification est douteuse, lors de mon prochain voyage au Mali en novembre, et vous l'apporterai (sic). [...]» (A.GD 10). 
Figure 10 : Lettre adressée à Roland Portères en 1973 témoignant de la permanence des relations de Germaine Dieterlen avec les membres du laboratoire d'ethnobotanique. La lettre se trouve dans la boite de carpothèque

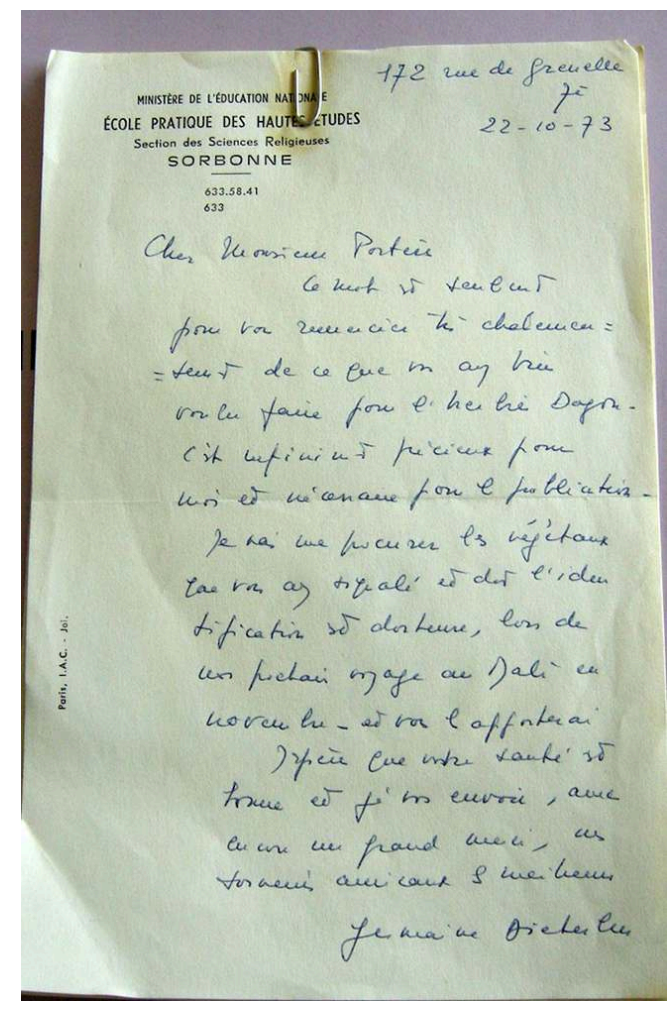

Photographie D. Juhé-Beaulaton

À la même époque, le Bureau de prospection des experts du Service de coopération technique internationale du ministère des Affaires étrangères adresse au CNRS une demande (non datée) d'experts "ayant une expérience d'une dizaine d'années au moins dans leur spécialité pour effectuer des missions temporaires dans les pays en voie de développement.[...] Ces missions peuvent intéresser des professeurs ou maîtres de conférence sur le point de prendre leur retraite et ayant conservé une activité physique leur permettant de supporter de nouveau climat » (A.GD 20). Cette demande, retrouvée dans les archives de G. Dieterlen, l'a certainement intéressée. Elle était alors à un tournant de sa vie - retraitée depuis 1973 à l'âge de 70 ans - et correspondait bien au profil de l'annonce. Elle avait déjà manifesté à la fin des années 1940 son implication dans les questions de développement et de santé par ses multiples enquêtes sur les plantes médicinales ${ }^{50}$. Si aucune archive n'indique que c'est par ce Bureau que G. Dieterlen a été contactée par le laboratoire pharmaceutique Debat ${ }^{51}$, divers documents figurant dans le même carton révèlent qu'elle se tourne à la même époque vers une anthropologie appliquée en collaborant avec ce laboratoire. Une lettre datée du 8 juillet 1976 de Leguen, directeur général des laboratoires Debat, relate un échange au sujet de plantes africaines lors de sa récente visite au domicile de G. Dieterlen, lettre accompagnée d'une liste des maladies susceptibles de les intéresser. Leguen précise que le service médical du laboratoire est chargé d'établir une description des symptômes de chacune de ces maladies afin de les identifier et d'éviter « tout malentendu au cours de [leur] collaboration ». Il espère ainsi " la mise au point de nouvelles thérapeutiques » (A.GD 10).Le financement prévu - 10000 francs - lui permettait de poursuivre ses missions en pays dogon. Le contrat passé entre le Laboratoire Debat et G. Dieterlen 
définit les conditions de travail, de recueil de renseignements sur les plantes et de réserve sur l'exploitation qui pourra en être faite ; il est signé le 26 novembre 1976 et stipule dans son article $1^{\text {er }}$ :

«Madame Dieterlen partant pour le Mali à la fin du mois de décembre prochain, s'engage à recueillir en ce pays pendant son séjour le maximum de renseignements concernant les plantes médicinales utilisées dans ce pays et ayant une action préventive et curative dans les maladies suivantes : rhumatisme, maladie à virus, lithiase rénale, maladie parasitaire, abcès, douleur (par exemple douleur dentaire), sommeil, bilharziose, onchocercose, paludisme, dépression nerveuse.

Elle enverra mensuellement à Debat, sur des fiches signalétiques qui lui auront été remises, ces renseignements qui comporteront notamment, dans la mesure du possible, le nom scientifique du végétal, son nom vulgaire, sa dispersion et toutes ses caractéristiques botaniques et écologiques, (désignation géographique, zone climatique et altitude des lieux de récolte), parties utiles de la plante (fleur, fruit, graine, pédoncule, tige, racine, pied, chapeau, écorce de telle ou telle partie du végétal), son mode de préparation, son mode d'administration et les effets qui en sont attendus.

Pendant la durée de son séjour madame Dieterlen fournira à Debat les plantes qui auront été retenues par Debat, soit 1 à $2 \mathrm{~kg}$ des parties utiles de chacune d'elles pour permettre de procéder aux essais pharmacologiques ». (A.GD 10).

Les archives de G. Dieterlen révèlent ce qu'elle a pu réaliser correspondant à ce contrat: elle a recueilli en pays dogon beaucoup d'informations sur les plantes médicinales - ce qu'elle avait déjà fait bien avant sa collaboration avec Debat - en relation avec certaines maladies identifiées selon les indications précitées par le laboratoire Debat. Elle a effectivement recueilli des plantes à Sanga en juillet 1977 et les a envoyées à Debat, comme l'attestent son cahier de terrain de 1977 et un document d'archive dactylographié présentant dans un tableau son herbier des plantes médicinales dogon de la même année ${ }^{52}$. Celui-ci comporte les noms de plantes en langue vernaculaire et les identifications botaniques réalisées par H. Gillet au Muséum, les maladies et la thérapeutique associée (Figure 11). En revanche, rien ne transparaît sur les envois mensuels attendus par Debat et la qualité des informations écologiques et botaniques. 
Figure 11 : Tableau de l'herbier des plantes médicinales dogon de 1977. (A.GD 10)

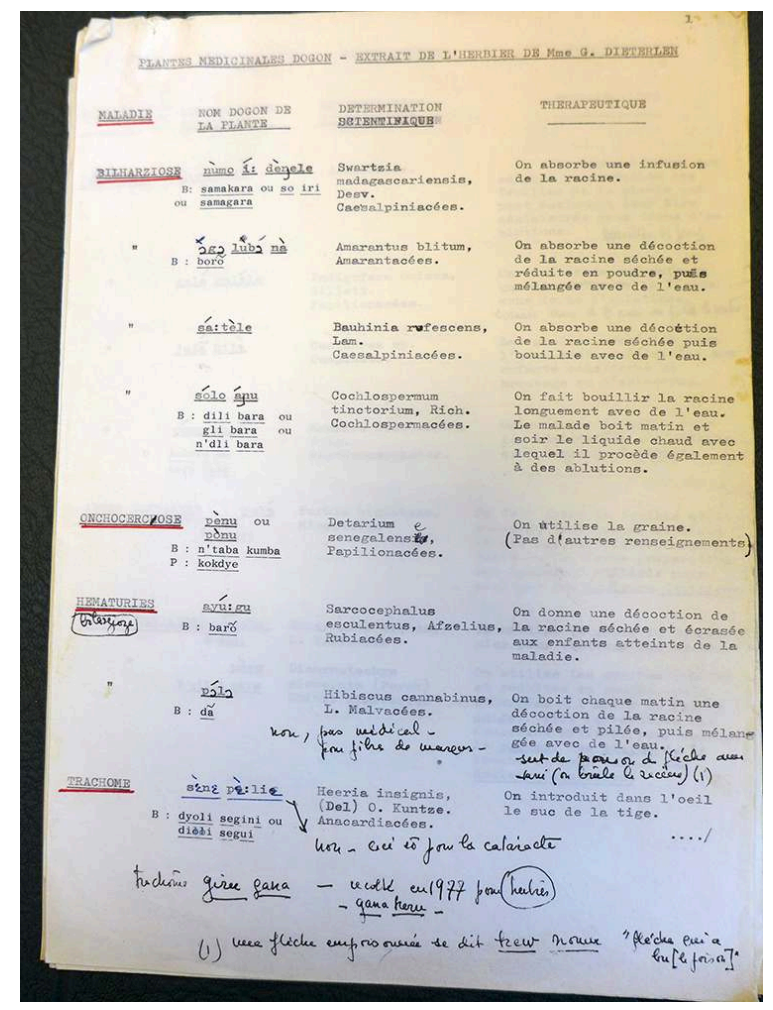

Photographie D. Juhé-Beaulaton

Comment a-t-elle procédé pour collecter herbiers et informations ? Si en Côte d'Ivoire elle n'a pas effectué de longs et nombreux séjours, elle a en revanche résidé régulièrement en pays dogon et suffisamment longtemps pour pouvoir recueillir ellemême les informations. Des notes dans ses cahiers de terrain attestent qu'elle était accompagnée par des guides-informateurs et que les collectes se faisaient sur plusieurs jours selon les dates indiquées dans ses carnets. Elle mentionne ainsi dans le carnet de 1973 le recueil de plantes et leurs usages à Sanga par Pangalé Dolo, fils de Ambara Dolo, décédé en 1971 ou $1972^{53}$. Le nom d'Amadigné Dolo, interprète, enquêteur et collecteur d'objets et de plantes, apparaît également comme informateur: elle note dans son cahier au sujet d'une plante appelée yamori qu'Amadigné « connaît cette plante pour le traitement de la dysenterie $»^{54}$.

Le plus souvent, des listes de noms de plantes suivis des usages figurent dans ses carnets. L'absence d'informations écologiques et botaniques qu'elle était censée relever selon les instructions du laboratoire Debat laisse supposer que ses informateurs lui ont apporté les échantillons des plantes qu'elle n'a donc probablement pas observées in $s^{i t u^{55}}$. Les informateurs ont peut-être voulu garder secret le lieu où se trouvaient les plantes dont certains usages également relevaient du secret, ce qui pose aussi la question des plantes choisies par les informateurs. Enfin, les distances à parcourir, parfois longues et dans des terrains accidentés peuvent avoir limité les observations ${ }^{56}$. Toujours est-il que, comme en Côte d'Ivoire, il semble que G. Dieterlen n'était pas toujours sur le terrain de la collecte avec ses informateurs. Une lettre de l'un d'eux, Ibrahima Guindo, en poste au musée de Bamako, nous apprend qu'il a reçu de l'argent pour l'envoi vraisemblable de plantes en mai 1976, avant la signature avec Debat. Il donne notamment des précisions sur les usages et le nom vernaculaire de l'une d'elles : 


\begin{abstract}
« bamako le 7-5-76
chere madame

Je vous envoie cette lettre pour vous donner mes nouvelles et recevoir les votres rien de mal jai recu votre lettre la contenance y comprise jair ecu l'arant merci : au sujet $\mathrm{du}$ fruit peu ou ntambacoumba en peulh ses concodiè sa soigne l'anchorcercose et le fironcle pour le fironcle vous écrasé et la poudre il faut mélanger avec l'eau et posé sur la plaie et ca fait des pus et pour l'anchorcercose vous brulé et la poudre vous mélangé avec la pommade à landroit ou ca vous fait mal vous frottrez au moment du coucher et le matin vous vous lavez il parait que ca soigne beaucous de maladie (sic). ".
\end{abstract}

En haut à droite figure une annotation manuscrite de G. Dieterlen «Roberty tambakumba - Detarium senegalensis - fruits leg. toxiques, peut-être identifié à partir du nom vernaculaire cité dans la Petite flore de l'Ouest-Africain de Guy Roberty (1954) dont le nom figure sur la lettre (A.GD 10) ${ }^{57}$. Cette plante se retrouve dans le tableau de l'herbier de 1977 et une nouvelle lettre d'I. Guindo, datée du 3 mai 1977 donne des informations complémentaires sur les utilisations médicinales de cette plante (A.GD 10) ${ }^{58}$. Une annotation « Debat » de G. Dieterlen figure en haut de la lettre.

« Je vous envoie cette lettre pour vous donner mes nouvelles et recevoir les vôtres. La réponse a duré j'étais en train de faire une enquête sur le pénu, en peulh c'est konkédié, en bambara c'est le tabacoumba.

$1^{\circ}$ ) Si on a du fironcle qui n'a pas pu faire du pû. On écrase on met un peu de sel mélangé avec un peu d'eau et mettre sur le fironcle.

$2^{\circ}$ ) Si on a tout le corps gonflet on écrase avec le " ganifie » en peulh c'est du gileton mélange avec ses deux avec du lait caillé on se frote avec tout le corps. Après avoir le corps séché là on peut se laver après.

$3^{\circ}$ ) ça soigne le sinejite si on a mal au fron ou le Céphalées on frote sur le fron.

$\left.4^{\circ}\right)$ ça soigne aussi un sein gonflé en mélange avec de l'eau fraiche le penu et frotté un peu le sein.

$\left.5^{\circ}\right)$ ça soigne aussi les dentitions des enfants. (sic)»

Un courrier daté du 2 août 1977 de Pierre Leguen, Directeur du laboratoire Debat, atteste la bonne réception de l'herbier collecté en juillet 1977. Il annonce à G. Dieterlen que les résultats des analyses pharmacologiques des échantillons lui seront envoyés (A.GD 10), résultats que l'on retrouve effectivement dans le même dossier. En décembre 1977, le laboratoire Debat a finalement retenu six plantes pour des essais pharmacologiques approfondis. Notons que le Detarium senegalensis n'y figure pas. L'Institut de recherches chimiques et biologiques appliquées (IRCEBA) de Beynes (78) est chargé des analyses, pour lesquelles il est demandé à Germaine de rapporter $3 \mathrm{~kg}$ de chacune d'entre elles (A.GD 10). En mars 1979, G. Dieterlen remet à Mr Guay du laboratoire Debat la quantité demandée de chaque plante sélectionnée comme indiqué dans son carnet de terrain de cette année-là ${ }^{59}$. 
Figure 12 : Plantes remises par G. Dieterlen au laboratoire Debat (mars 1979) (A.GD 10)

\begin{tabular}{c|c|c|c} 
Nom vernaculaire & Nom scientifique & Famille & Poids en kg \\
\hline$b i$ & Sclerocarya birrea (A.Rich.) Hochst & Anacardiacées & 10 \\
\hline$d i$ : lolorio & Heteranthera callifolia Reichb. ex Kunth & Pontederiacées & 3 \\
\hline$d i$ : bibile & Scoparia dulcis L. & Scrophulariacées & 3 \\
\hline iduolo & Aristida kerstingii Pilger & Poacées & 3 \\
\hline edegele & Sesbania punctata DC. & Papilionacées/Fabacées & 3 \\
\hline dogoku : ninu & Gynandropsis pentaphylla DC. & Capparidacées & 3
\end{tabular}

Le travail entrepris pour le laboratoire Debat donne l'occasion à G. Dieterlen de reprendre la classification des végétaux publiée en 1952 en intégrant cette fois-ci les plantes médicinales. Son intention était de publier une révision de la classification de 1952 augmentée des plantes médicinales auxquelles elle n'a cessé de s'intéresser. À nouveau, un brouillon de son article $e^{60}$, retrouvé dans ses archives, donne des informations précieuses sur la chronologie de ce travail et son mode de fonctionnement. Elle précise que l'herbier a été déposé au laboratoire d'ethnobotanique et les plantes identifiées par R. Portères, décédé en 1974, puis par H. Gillet. Elle remercie également Joan Bungener, assistante au laboratoire d'ethnobotanique, pour la dactylographie des listes et tableaux. Ce travail n'a pas été publié, semble-t-il, alors qu'il représente la synthèse de ses recherches sur les plantes médicinales et les savoirs associés qu'elle a pu recueillir. Comment expliquer une fois de plus l'inachèvement de cette étude aux frontières de l'ethnologie et de l'ethnopharmacologie? Le devoir de réserve exprimé dans le contrat passé entre G. Dieterlen et le laboratoire Debat peut éventuellement le justifier. Cependant, le problème récurrent des difficultés d'identification des échantillons d'herbier se retrouve dans de nombreux documents et peut expliquer à lui seul cet échec: le botaniste J.G. Adam (1909-1980), sollicité par J. Bungener au sujet de plantes difficiles à identifier, évoque dans une lettre des mélanges d'herbier sur les feuilles et conseille de contacter le botaniste Laurent Aké Assi en Côte d'Ivoire ${ }^{61}$.

De T. Monod en 1954 à l'Ifan à R. Portères et H. Gillet au Muséum, les botanistes ont très souvent estimé la qualité de nombre d'échantillons de plantes récoltées par G. Dieterlen - ou plutôt par ses informateurs - trop médiocre d'un point de vue botanique pour permettre leur identification scientifique (échantillon mal conservé, incomplet). Or, celle-ci constitue le premier acte indispensable à toute étude pharmacologique ou ethnobotanique. Notons que pour les informateurs et collecteurs des échantillons, ces derniers étaient de qualité suffisante pour transmettre les noms vernaculaires et leurs savoirs. En outre, la manipulation des plantes lors des entretiens après la collecte a pu les abimer ${ }^{62}$ et générer des pertes alors même que le passage du vivant à l'échantillon sec comporte également des risques. 


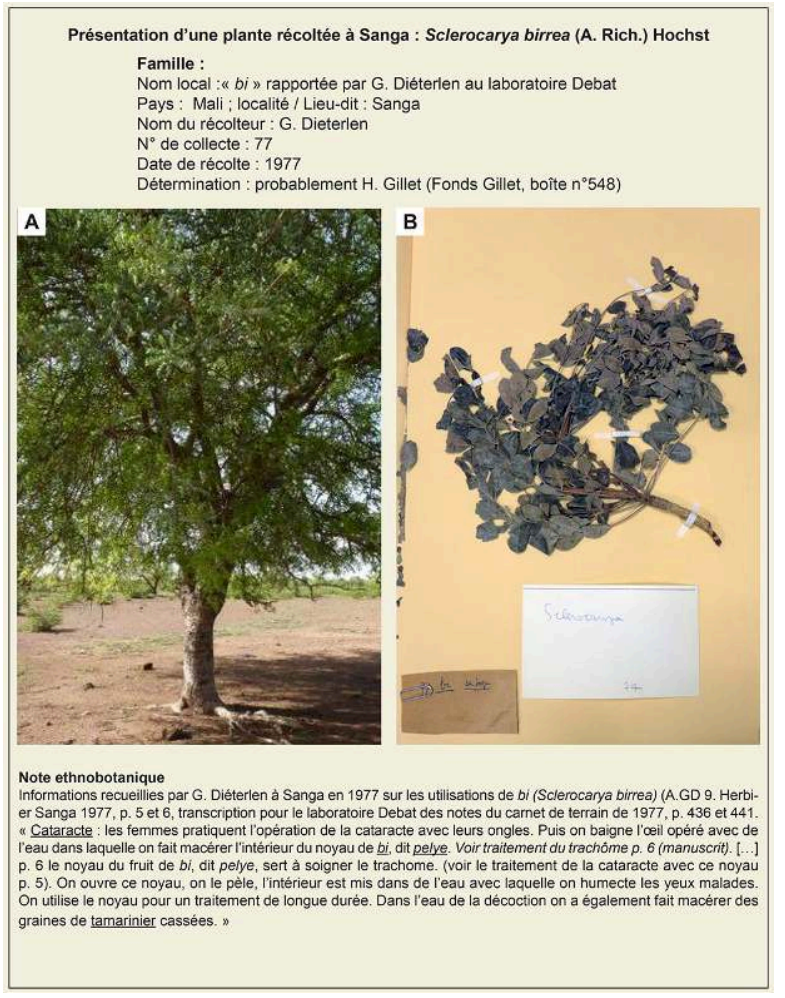

A :Sclerocarya birrea(A. Rich.) @ C. Pirat, https://api.tela-botanica.org/img:0003406440.jpg

B : Échantillon de bi, Sclerocarya birrea (A. Rich.) Hochst, provenant du Mali, probablement déterminé par H. Gillet. Il a été retrouvé en juin 2019 dans la boîte n 548 de la collection Gillet. @ D. JuhéBeaulaton

\section{Conclusion}

G. Dieterlen a mis à profit toutes les occasions offertes au cours de ses missions pour faire des enquêtes sur les plantes médicinales. Ses travaux de recherche les plus aboutis mobilisant l'ethnobotanique restent les plantes ichtyotoxiques (1952a), la classification dogon des végétaux (1952b) et l'alimentation avec G.Calame-Griaule (1960). Ses rapports d'activités révèlent un décalage entre la réalité du terrain et l'annonce des résultats escomptés. Les données accumulées trouvées dans ses archives et herbiers, non publiées, montrent la différence entre la volonté ou le désir de faire et les contraintes ou limites imposées par les conditions du terrain (conservation des échantillons d'herbier en milieu tropical, relations avec les informateurs...), celles posées par les éditeurs (texte trop long, volume trop important de données pour Bregbo) et interroge les activités de tout scientifique dont les recherches parfois n'aboutissent pas.

Plusieurs documents mentionnent les problèmes posés par l'identification des plantes récoltées en raison de l'insuffisance des échantillons : la mauvaise qualité des herbiers de G. Dieterlen est une caractéristique fréquente des herbiers d'ethnologues. Ceci tient à leur absence de formation initiale en botanique et technique d'herborisation ${ }^{63}$. Si Julien Bondaz(2018 : 364-365) voit dans la pratique de l'herbier par les ethnologues de cette époque une forme de "légitimation scientifique», je pense néanmoins que la détermination botanique des plantes citées par les informateurs est bien indispensable 
pour permettre d'établir une concordance entre les systèmes locaux de classification et la taxonomie botanique. Un nom vernaculaire de plante ne peut avoir son correspondant botanique que par le recours à l'herbier et seule la taxonomie scientifique permet une véritable réflexion sur les systèmes de classification locaux. G. Dieterlen l'avait bien compris et l'impossibilité d'identifier nombre d'échantillons peut expliquer en partie l'échec de la publication sur les plantes de Bregbo et celui de la révision de sa classification de $1952^{64}$.

Cette étude apporte aussi un éclairage sur le rôle des informateurs locaux qui s'investissent dans les recherches. Il ne s'agit pas ici de critiquer les méthodes de terrain de G. Dieterlen, mais il semble bien que la récolte des échantillons ait été dévolue à ses informateurs privilégiés, ce qui explique l'apport d'échantillons de plantes déconnectées de leur contexte écologique ou de celui de leur production et dont la mauvaise qualité tient autant aux circonstances de la collecte qu'à la méconnaissance des techniques d'herborisation transmise par une ethnologue ellemême peu formée. Il faut aussi distinguer le temps de l'enquête de celui de la collecte. Si le botaniste procède par relevés des espèces végétales et prélèvement d'échantillons pour une identification et un conditionnement ultérieurs, l'ethnologue réalise des entretiens difficiles à mener en parallèle de la collecte d'échantillons. Celle-ci peut précéder l'entretien comme semblent l'attester les cahiers de terrain de G. Dieterlen. La durée d'un entretien, variable, peut être de plusieurs heures et le temps de conditionnement de l'herbier réuni peut s'en trouver raccourci. En zone tropicale, il est nécessaire d'assurer un bon séchage des échantillons et pour cela il faut changer très régulièrement les papiers et mettre l'herbier à sécher dans de bonnes conditions, opérations fastidieuses et dispendieuses en temps. Ces différences de traitement peuvent expliquer la mauvaise conservation des herbiers de certains ethnologues dont quelques-uns sont gardés dans les collections d'ethnobotanique du Muséum ${ }^{65}$.

Cependant, si leurs échantillons ne sont pas intéressants d'un point de vue botanique, ils permettent néanmoins l'identification des plantes utilisées par les sociétés étudiées et apportent des informations linguistiques et culturelles. En outre, le progrès des analyses génétiques laisse envisager de nouvelles potentialités, même à partir d'échantillons de mauvaise qualité. Les savoirs botaniques associés aux plantes apportent des informations complémentaires sur leur origine et donc leurs circulations y compris celles des humains, sur leur écologie et donc sur les changements environnementaux... Si les herbiers peuvent contribuer à mesurer l'érosion de la biodiversité, ceux des ethnologues revisités pourraient également permettre d'évaluer celle de la diversité culturelle et des savoirs locaux, si tant est que l'application du protocole de Nagoya (2010) ne limite pas ce type de recherche ${ }^{66}$. Un point doit être souligné à ce sujet: le devenir de la collection de G. Dieterlen et des informations d'ordre linguistique et culturel qu'elle contient s'inscrit dans le cadre de la Convention sur la diversité biologique (1992) qui recommande "le rapatriement» des informations, y compris des «connaissances autochtones et traditionnelles» (Bahuchet et al. 2019), préconisation renforcée par le protocole de Nagoya.

Enfin, ces herbiers étudiés comme des sources par les historiens éclairent d'un nouveau regard l'histoire des sciences humaines. Cet article s'inscrit ainsi dans ce nouvel intérêt porté aux collections. En effet, l'échantillon de plante est aussi un objet ou une image sur lequel se construit la démarche intellectuelle de G. Dieterlen dont il représente la matérialité au même titre que ses notes de terrain soigneusement conservées dans des 
cahiers classés chronologiquement dans ses archives. L'herbier apparaît comme un instrument du travail scientifique de l'ethnologue et offre une histoire matérielle de cette discipline qui reste encore à explorer, dans la prolongation des travaux sur la matérialité des savoirs de F. Waquet $(2015)^{67}$.

\section{BIBLIOGRAPHIE}

Augé M., Bureau R. \& Piault C. (Ed.) 1975 - Prophétisme et thérapeutique : Albert Atcho et la communauté de Bregbo. Paris, Hermann, 332 p.

Bahuchet S. 2018 - Claudine Friedberg (1933-2018). Revue d'ethnoécologie [En ligne], 14, mis en ligne le 31 décembre 2018. URL : http://journals.openedition.org/ethnoecologie/3798 ; DOI : 10.4000/ethnoecologie.3798

Bahuchet S. \& Lizet B. 2001 - L'ethnobotanique au Muséum national d'histoire naturelle. Les hommes, les idées, les structures. In : Lieutaghi P. \& Musset D. (Ed), Plantes, sociétés, savoirs, symboles. Matériaux pour une ethnobotanique européenne. Mane, Musée-conservatoire de Salagon et Les Alpes de lumière : 15-32. (Les cahiers de Salagon ; 8).

Bahuchet S., Blanc J., Hoare C., Juraver S., Kourdourli M. \& Pennec F. 2019 - Des hommes et des plantes. Revue d'ethnoécologie [En ligne], 16 | 2019, mis en ligne le 31 décembre 2019, URL : http:// journals.openedition.org/ethnoecologie/5786 ; DOI : https://doi.org/10.4000/ethnoecologie.5786 Bondaz J. 2016 - Ethnobotanique. À la naissance de l'ethnologie française. Les missions ethnographiques en Afrique subsaharienne (1928-1939). [En ligne] URL : http://naissanceethnologie.fr/exhibits/show/ ethnobotanique

Bondaz J. 2018 - De l'herbier à l'arbre cosmique. L'École Griaule et l'ethnobotanique (1926-1965). In : Juhé-Beaulaton D. \& Leblan V. (Ed.), Le spécimen et le collecteur. Savoirs naturalistes, pouvoirs et altérités (XVIII'-XX siècle). Paris, MNHN : 349-383. (Archives ; 27).

Boulvert Y. 2011 - Hubert Gillet (1924-2009). Ethnobiologiste du désert. In : Serre J. (Ed.) Hommes et destins : Tome 11. Afrique noire. Paris : Académie des Sciences d'Outre Mer ; L'Harmattan : 349-351.

Bouquet A. \& Debray M. 1974 - Plantes médicinales de la Côte d'Ivoire. Paris, Office de la recherche scientifique et technique outre-mer (O.R.S.T.O.M.), 231 p. (Travaux et documents ; 32).

Bourguet M.-N. 2010 - A portable world: the notebooks of European travellers (eighteenth to nineteenth centuries). Intellectual History Review 20 (3) : 377-400.

Brousse C. 2017 - De l'objet à caractère végétal à l'objet ethnobotanique. Penser les collectes de plantes et objets en plante. In Situ [En ligne], 32. URL : http://journals.openedition.org/insi- tu/ 15414 ; DOI : 10.4000/insitu.15414

Chadefaud M. 1979 - Portrait de Roger Heim. Revue de Mycologie 43 : 323-329.

Chevalier A. 1937 - Les plantes magiques cultivées par les Noirs d'Afrique et leur origine. Journal de la Société des Africanistes 7 (1) : 93-105. 
Crémieux Brilhac J-L. 1995 - Henri Laugier en son siècle. CNRS Editions. (Cahiers pour l'histoire de la recherche). [En ligne], http://www.histcnrs.fr/laugier-chr.html

Delpuech A. 2017 - Collectes, collecteurs, collections dans les années trente : entre théorie ethnologique et réalités pratiques. In : Delpuech A., Laurière C. \& Peltier-Caroff C., Les Années folles de l'ethnographie. Trocadéro 28-37. Paris, Muséum national d'Histoire naturelle : 449-479. (Archives ; 25).

Dieterlen G. 1952a - Emploi de plantes ichtyotoxiques chez les Dogon de Sanga. Notes Africaines $56: 113-116$.

Dieterlen G. 1952b - Classification des végétaux chez les Dogon. Journal de la Société des Africanistes $22(1): 115-158$

DieterlenG. \& Calame-Griaule G.1960 - L'alimentation dogon. Cahiers d'études africaines 1 (3) : 46-89.

Doquet A. 2009 - Le terrain des notes. Enquête, notes de terrain et raisonnement de l'anthropologue. Langage et société 127 (1) : 52-70.

Fischer G. \& Laugier H.1960 - Pour une Université internationale au service des pays sousdéveloppés. In : Tiers-Monde, La planification de l'éducation et ses facteurs économiques et sociaux. Colloque international de Paris (9-18 décembre 1959) 1(1-2) : 17-26.

Friedberg C. 1974 - Les processus classificatoires appliqués aux objets naturels et leur mise en évidence. Quelques principes méthodologiques. Journal d'agriculture tropicale et de botanique appliquée 21 (10-12) : 313-334.

Ganay S. de 1942 - Le xylophone chez les Sara du Moyen Chari. Journal de la Société des Africanistes $12: 203-240$.

Ganay S. de 1947 - Un jardin d'essai et son autel chez les Bambara. Journal de la Société des Africanistes 17 : 57-63.

Griaule M. \& Leiris M. 1931 - Instructions sommaires pour les collecteurs d'objets ethnographiques. Paris : Musée d'ethnographie. (Mission scientifique Dakar-Djibouti).

Griaule M. 1947 - Le verger des Ogol (Soudan Français). Journal des Africanistes 17 (1) : 65-79.

Griaule M. 1957 - Méthode de l'ethnographie. Paris, Presses universitaires de France, 107 p.

Hellweg F. L. 2019 - Les défis de la prise en charge des personnes atteintes des maladies mentales en médecine traditionnelle en Côte d'Ivoire. Quel positionnement des psychothérapeutes traditionnels? Une recherche anthropologique au Centre Thérapeutique Atcho à Bregbo. Mémoire de Master 2, Expertise en Population et Développement, Université Paris Descartes, $103 \mathrm{p}$.

Heusch L. de 1975 - Avant-propos. Systèmes de pensée en Afrique noire 1 : 3-4.

Heusch L. de 2001 - Ma dette de reconnaissance envers Germaine Dieterlen. Journal des africanistes 71 (1) : 69-75.

Jolly É. 2001 - Marcel Griaule, ethnologue : la construction d'une discipline (1925-1956). Journal des africanistes 71 (1). Les empreintes du renard pâle : 149-190.

Jolly É. 2016a - Germaine Dieterlen. In : À la naissance de l'ethnologie française. Les missions ethnographiques. http://naissanceethnologie.fr/exhibits/show/germaine_dieterlen

Jolly É. 2016b - Amadigné Dolo. In : À la naissance de l'ethnologie française. Les missions ethnographiques. http://naissanceethnologie.fr/files/pdf/101.pdf 
Jolly É. \& Lemaire M. (Ed.) 2015 - Cahier Dakar-Djibouti. Meurcourt, Éditions Les Cahiers, 1408 p. Laugier H. 1966 - Message d'Henri Laugier. Tiers-Monde 7 (28), Désarmement et développement : 667-670.

Laurière C. 2008 - Paul Rivet : le savant et le politique. Paris, Muséum national d'Histoire naturelle, 723 p. (Archives ; 12).

Lebrun A. 1937 - Mission de S. de Ganay et G. Dieterlen chez les Dogon (Soudan français). Journal de la Société des Africanistes 7 (2) : 227-233.

Lemaire M. 2011 - La chambre à soi de l'ethnologue. Une écriture féminine en anthropologie dans l'Entre-deux-guerres. L'Homme 200 : 83-112.

Mauss M. 1947 - Manuel d'ethnographie (notes rassemblées par Denise Paulme à partir des cours de Mauss à l'institut d'ethnologie). (Classiques UQAC).

Piault M.H. 2001 - Avant-propos. Germaine Dieterlen et la « parole claire ». Journal des africanistes 71 (1), Les empreintes du renard pâle : 7-16.

Portères R.1957 - Vers une Organisation française de la Recherche Scientifique et Technique sur de nouvelles drogues pharmaceutiques d'origine végétale. Journal d'agriculture tropicale et de botanique appliquée 4 (1-2) : 94-105.

Roberty G. 1954 - Petite flore de l'Ouest-Africain. Paris, ORSTOM, 441 p.

Rouch J. 1963 - Introduction à l'étude de la communauté de Bregbo. Journal des Africanistes 33 (1) : 129-202.

Rouch J. 1975 - En diable. In : Piault C., Bureau R. \& Augé M. (Ed.), Prophétisme et thérapeutique : Albert Atcho et la communauté de Bregbo. Paris, Hermann : 11-26.

Waquet F. 2015 - L'ordre matériel du savoir. Comment les savants travaillent (XVI ${ }^{\mathrm{e}}-\mathrm{XXI} \mathrm{e}^{\mathrm{e}}$ siècles). Paris, CNRS Éditions, 359 p.

Webographie

GBIF / Global Biodiversity Information Facility :https://www.gbif.org/species/3171787

Prota4uweb data de Wageningen University : https://www.prota4u.org/database/protav8.asp?

fr=1\&g=pe\&p=Sclerocarya+birrea+(A.Rich.)+Hochst ; https://www.prota4u.org/database/ protav8.asp?fr=1\&g=pe\&p=Detarium+senegalense+J.F.Gmel ; https://www.prota4u.org/database/ protav8.asp?h=M4\&t=Scoparia,dulcis\&p=Scoparia+dulcis\#Synonyms

Société Française d'Ethnopharmacologie. Plantes médicinales et pharmacopées traditionnelles :http:// www.ethnopharmacologia.org/recherche-dans-prelude/?plant_id=5317\#lightbox[5317]/0/

Telabotanica :https://www.tela-botanica.org/thematiques/flora-data/

\section{ANNEXES}

Annexe 1 : Herbiers et archives de Germaine Dieterlen

L'herbier Dieterlen conservé au MNHN est composé de six boîtes numérotées ( $n^{\circ} 173$ à 178) contenant 461 parts d'herbier dont 154 ont pu être identifiées, une boîte $n^{\circ} 1521$ (initialement déposée à l'herbier) contenant 99 parts et une boîte de carpothèque $\left(n^{\circ} 243\right)^{68}$. Dans les boîtes $n^{\circ} 175,176,178$ et 1521 se trouve la série de parts d'herbier «Pharmacopée Bregbo », les deux autres boîtes $(173,174)$ et la carpothèque 
contiennent des parts d'herbier venant de diverses missions, principalement du pays dogon. Lors d'un reconditionnement partiel réalisé en 2016 (projet OMUSHIS financé par Sorbonne université), la boite $\mathrm{n}^{\circ} 177$ manquant de contenu a été intégrée à la boite $\mathrm{n}^{\circ} 173$.

Des parts de son herbier ont également été retrouvées dans quatre boîtes du fonds Gillet (boîtes $n^{\circ} 548$ à 552). L'intégration en cours des collections ethnobotaniques à l'Herbier général va entraîner leur reconditionnement et ces cotes vont donc évoluer.

Figure 14 : Tableau récapitulatif des herbiers et archives de Germaine Dieterlen consultés

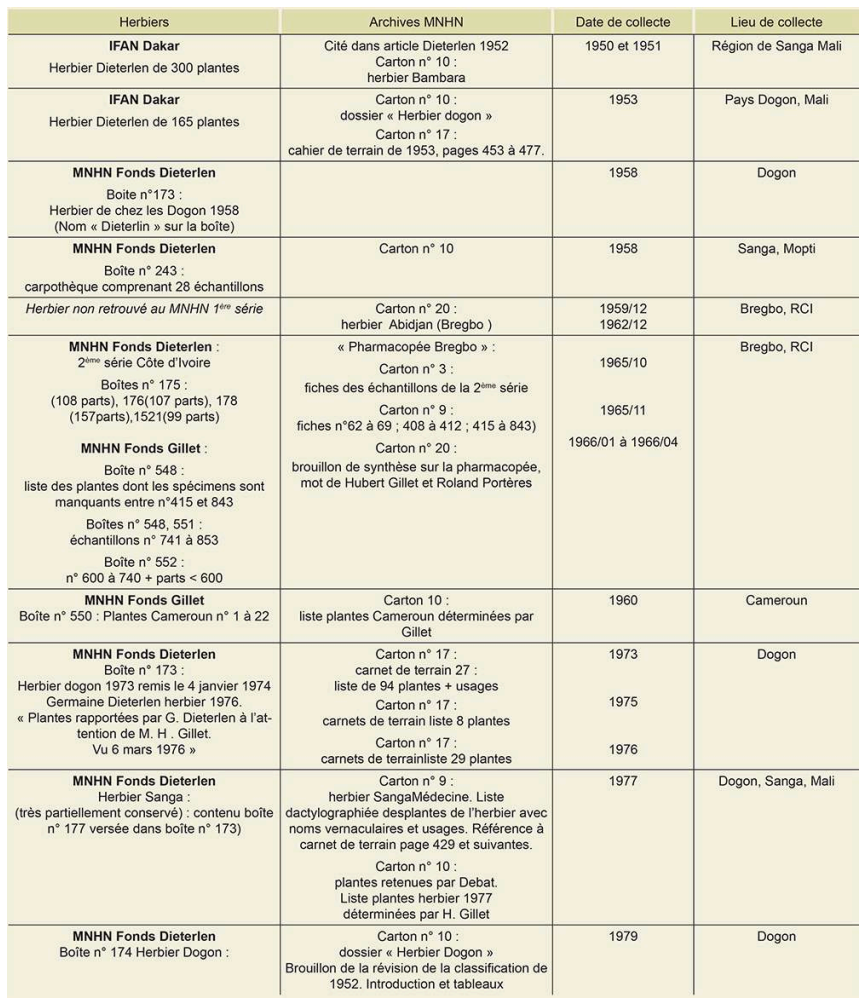

La carpothèque (boite $n^{\circ} 243$ ) comprend en tout 28 échantillons principalement de graines (céréales, légumineuses...) et des fiches comportant leur identification botanique et des informations ethnographiques (noms vernaculaires, usages) provenant des fiches de collecte de G. Dieterlen (Figure 15). L'écriture a pu être identifiée comme étant celle de Roland Portères. Cette carpothèque comprend les échantillons suivants :Vigna unguiculata (haricot dolique, niébé) (2), maïs Zea mays (1) sorgho (Sorghum margaritiferum (3), Sorghum caudatum (2), Sorghum cernuum (1), Sorghum guineense (1), Digitaria exilis (2), Sesamum indicum (1), Hibiscus sabdarifa (1) collectés par G. Dieterlen en 1958 à Sanga près de Bandiagara (Mali) et sept échantillons de différentes variétés de riz (Oryza glaberrima) provenant de Mopti (1958). Roland Portères a intégré dans son importante collection de riz des échantillons rapportés par G. Dieterlen. Il leur a attribué un nouveau numéro (8074) tout en conservant celui de la fiche de collecte de G. Dieterlen (Fig. Dieterlen 6490) (Figure 16). 
Figure 15 : Boîte de carpothèque. Collection ethnobotanique Fonds Dieterlen

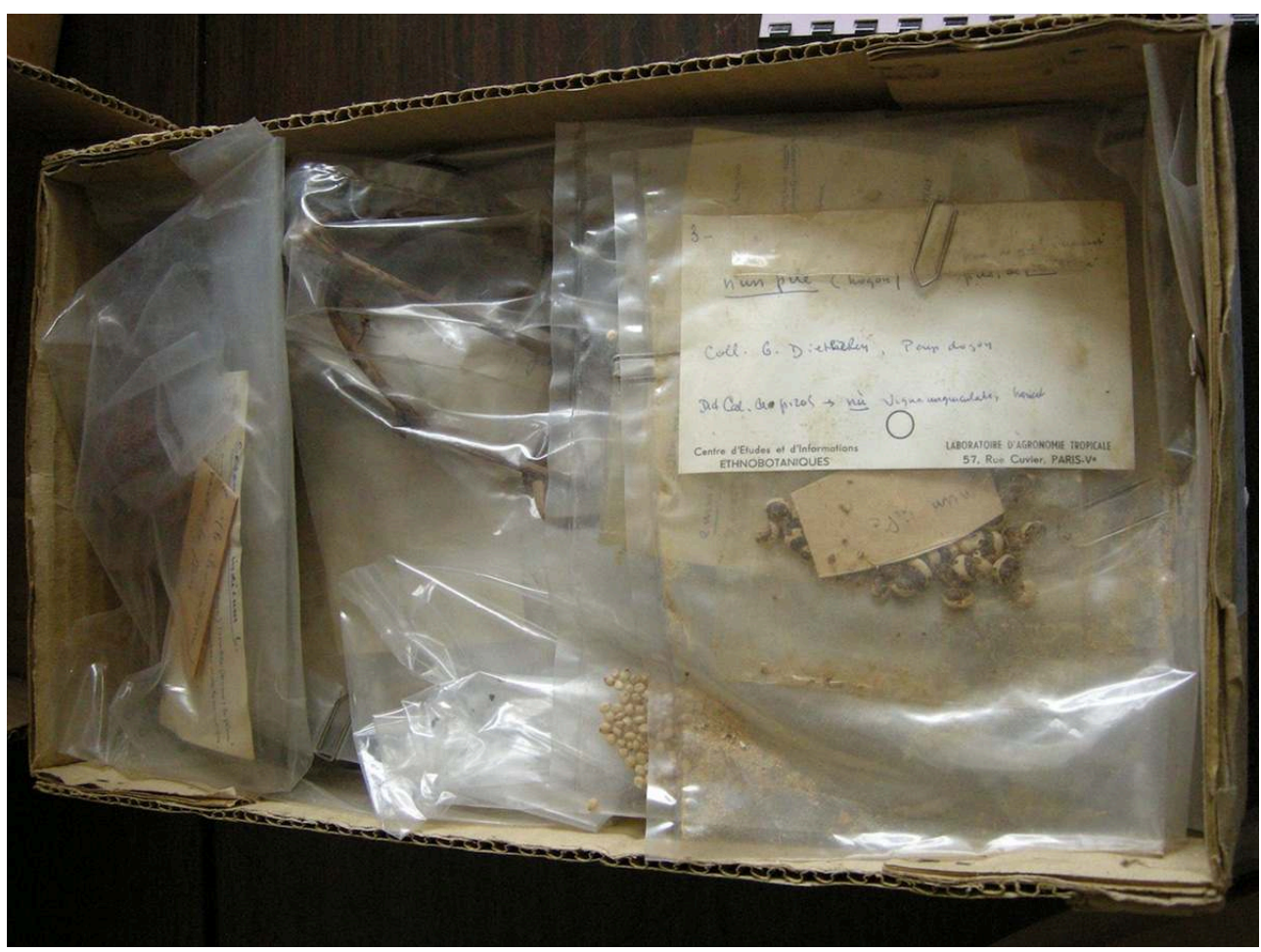

Photographie S. Juraver

Figure 16 : Échantillon de riz rapporté par G. Dieterlen versé à la collection Portères avec un nouveau numéro (8074) tout en conservant celui (6490) de la fiche de collecte de G. Dieterlen

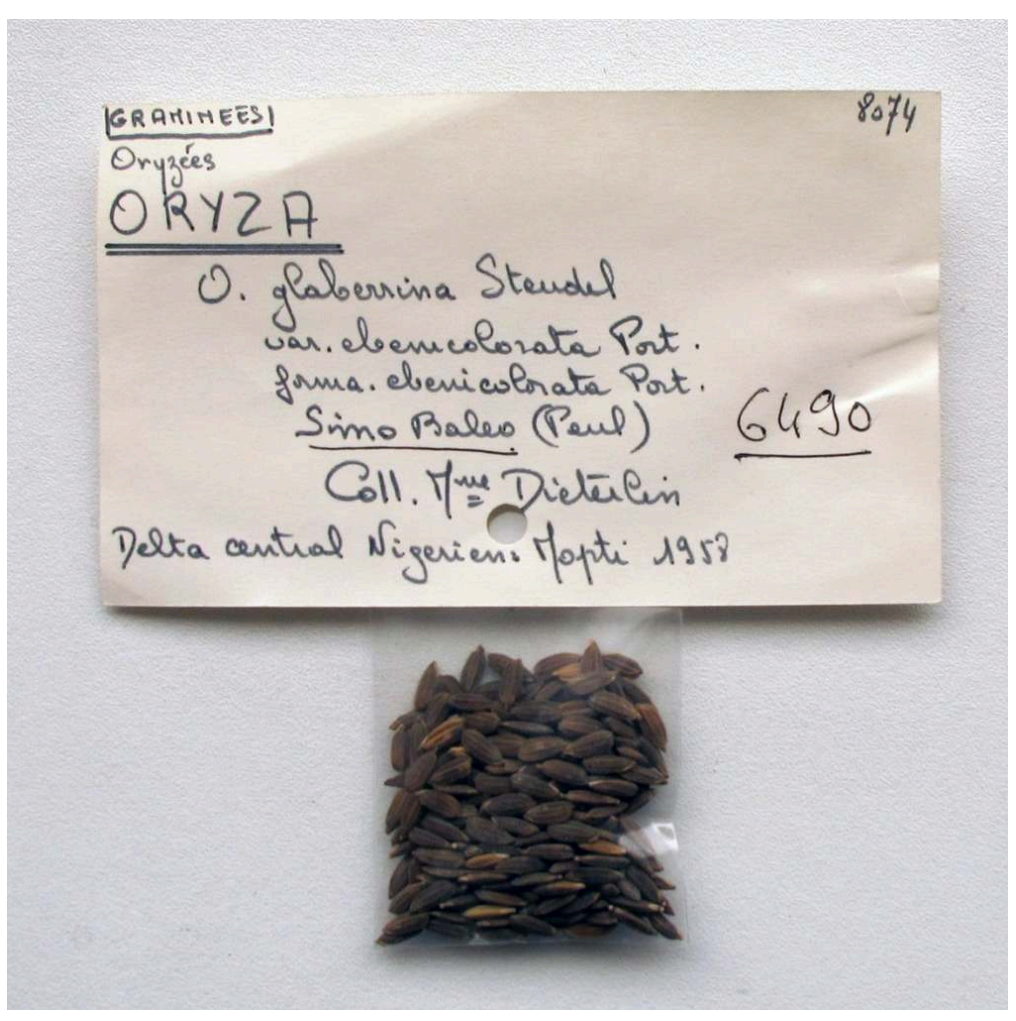

Photographie S. Juraver 
Annexe 2 : Brouillon d'un article de G. Dieterlen

« Dans un article publié en 1952, nous présentions les premiers résultats de recherches menées sur les végétaux sauvages et leurs usages chez les Dogon du Mali, en même temps qu'était constitué un herbier. Les enquêtes ont fait apparaître une classification dogon des végétaux que nous avons commentée. Depuis cette publication, nous avons régulièrement poursuivi la collecte des échantillons et de leurs divers usages. Dans la présentation des documents qui font l'objet de cet article, nous avons repris la liste des végétaux précédemment publiés pour y apporter corrections et adjonctions et intégrer les noms et ce que nous avons étudiés depuis 1952.

Nous donnons ici cette nouvelle liste, présentée comme la précédente selon le système classificatoire des Dogon. Nous rappelons que toutes les classifications de cette population sont mises en correspondances les unes avec les autres, ces associations étant entièrement tributaires du mythe cosmogonique sur lequel s'appuient toutes les croyances et les institutions. En ce qui concerne les liens avec la mythologie, nous renvoyons le lecteur à notre publication de 1952 dans laquelle elle a été presque complètement exposée. En revanche les informations sur l'usage médical des végétaux, systématiquement recueillies, sont ici toutes intégrées, elles sont très nombreuses. Leur collecte fait partie d'une enquête menée parallèlement sur les guérisseurs Dogon - dont nous avions parlé succinctement dans notre précédente publication - sur leurs techniques, leur rôle social, leurs croyances et leurs rites, enquête dont les résultats font actuellement l'objet d'un article en cours de rédaction. Les noms vernaculaires et les termes dogon sont transcrits selon le système phonétique adopté par madame Calame-Griaule dans son dictionnaire Dogon paru en 1968.

Tous les végétaux ont été déposés au laboratoire d'ethnobotanique du Muséum d'histoire naturelle de Paris et déterminés grâce à l'obligeance du regretté professeur Portères et du professeur Gillet que nous remercions de leur collaboration.

La présentation de ce document a été réalisée avec le concours assidu de madame Joan Bungener. Elle nous a secondé pour le dépouillement des informations recueillies qui ont été intégrées dans les classifications dogon précédemment établies. Elle a réalisé un index des noms scientifiques et un répertoire des affections mentionnées. Nous la remercions de sa collaboration. » (A.GD 10).

\section{NOTES}

1. Directrice de recherche au CNRS puis directrice d'études à la $\mathrm{V}^{\mathrm{e}}$ section (sciences religieuses) de l'École pratique des hautes études où elle était responsable du groupe des chercheurs africanistes se consacrant à l'étude des religions (1969-1973). En 1974, reconnaissant les travaux de ce groupe, l'EPHE décide la création du laboratoire associé au CNRS « Systèmes de Pensée en Afrique Noire » dont la direction est confiée à M. Cartry et L. de Heusch (Heusch 1975).

2. Les archives de G. Dieterlen, initialement déposées au Musée de l'Homme, ont été transférées après sa fermeture pour rénovation à la Bibliothèque centrale du MNHN en 2009. Le préinventaire du fonds permet d'identifier les cinq cartons - sur un total de 25-où se trouvent les documents sur les herbiers. Dans cet article, le terme "boîte» est employé pour les parts d'herbier et celui de "carton » pour les archives. Celles-ci seront citées ainsi : A.GD 3 (carton $\mathrm{n}^{\circ} 3$ ). Voir Annexe 1 pour la présentation des herbiers et des cartons d'archives associés.

3. Sur l'histoire de l'ethnobotanique, voir Bahuchet et Lizet (2001), Bahuchet et al. (2019),Bondaz (2016 et 2018). 
4. La mission, financée par l'État français, compte une équipe de dix personnes, des linguistes, des ethnographes, un musicologue, un peintre et un naturaliste, dirigée par M. Griaule et commanditée par P. Rivet et G.-H. Rivière, alors directeur et sous-directeur du Musée d'ethnographie du Trocadéro. Voir E. Jolly et M. Lemaire (2015).

5. P. Rivet (1876-1958), médecin militaire de formation et fondateur du Musée de l'homme, a luimême recueilli des échantillons botaniques lors de ses missions en Amérique latine dès 1902 déposés au MNHN : 219 plantes de Quito en 1902 (p. 10, vol. 3 du registre des entrées), 342 plantes d'Équateur en 1903 (p. 13-15-16), 209 en 1904 (p. 22)... (https://science.mnhn.fr/catalogue/botaentrees-vol3-1901-1947/). Notons l'influence de P. Rivet dans l'application du modèle naturaliste dans les collectes d'objets considérés comme ou sur le modèle du spécimen (Delpuech 2017 : 455 ; voir aussi Laurière 2008 : 63.).

6. Le Manuel d'ethnographie de M. Mauss, rédigé à partir des notes prises par les étudiants ayant assisté à ses cours entre 1926 et 1939, a été édité par Denise Paulme en 1947. (Voir Mauss 1947 : 45).

7. Instructions publiées à l'occasion de la mission Dakar-Djibouti et dont la rédaction est attribuée à M. Leiris (1901-1990) (Jolly \& Lemaire 2015). G. Calame-Griaule publie le cours de M. Griaule, Méthode de l'ethnographie, en 1957.

8. Registre des entrées, vol.3, p. 204. https://science.mnhn.fr/catalogue/bota-entreesvol3-1901-1947/

9. Registre des entrées, vol. 3, p. 246 et 249. Certains échantillons de l'herbier de S. de Ganay comportent des informations linguistiques, botaniques et parfois même écologiques sur une fiche collée. Voir comme exemple l'échantillon de Cola cordifolia (Spécimen P00516663 MNHN, Paris (France). Coll.: Plantes vasculaires (P); http://coldb.mnhn.fr/catalognumber/mnhn/p/ p00516663

10. Ce qui explique le dépôt de S. de Ganay au MNHN. Griaule (1947) a utilisé son travail pour son article sur les vergers des Ogol.

11. Les femmes ethnologues étaient encouragées à cette époque à partir sur le terrain et soutenues dans leurs demandes financières par les responsables de l'Institut d'ethnologie et le Musée d'ethnographie. Voir Lemaire (2011) sur la spécificité des missions des femmes ethnologues.

12. A.GD 10 : questionnaire et brouillon de l'article sur les plantes ichtyotoxiques (Dieterlen 1952a). J. Bondaz, qui a consulté les archives à l'IFAN-CAD (2018: 364 note 48), mentionne aussi une lettre de mars 1952 avec liste de déterminations botaniques de A. Pitot, Directeur du Département de botanique à l'IFAN, à G. Dieterlen.

13. $S$. de Ganay ne se réfère pas à des identifications botaniques dans ses publications (les espèces utilisées pour la fabrication du xylophone chez les Sara du Moyen Chari ni celles cultivées dans le jardin d'essai des Bambara (Ganay 1942 et 1947) alors que ses échantillons conservés au MNHN sont bien renseignés. Voir supra note 9.

14. Dieterlen $1952 b$ et rapport de mission de 1953, A.GD carton 20. Théodore Monod à l'IFAN encourageait les ethnologues à contribuer par leurs collectes à la connaissance de la flore africaine. Voir J. Bondaz(2018).

15. D'après J. Bondaz (2016), elle a pu utiliser sans la citer les travaux antérieurs de Solange de Ganay. Cependant S. de Ganay et G. Dieterlen se sont retrouvées à plusieurs reprises à Sanga et cette dernière s'appuie sur ses propres collectes déterminées à l'IFAN. L'herbier de S. de Ganay conservé au MNHN a été déterminé tardivement, à différents moments entre 1957 et 1972 et celui qui est conservé à la Bibliothèque Eric de Dampierre à l'Université de Nanterre est resté non monté et non déterminé.

16. Voir E. Jolly (2016b) sur les interprètes et informateurs des ethnologues. Ceux-ci ont commencé à travailler lors de la première mission Griaule, souvent de jeunes écoliers recommandés par l'école de Sanga. 
17. Auguste Chevalier, premier directeur du laboratoire d'agronomie coloniale du MNHN s'est beaucoup intéressé aux plantes utiles et aux savoirs locaux. Il est considéré comme le précurseur de l'ethnobotanique en France (Bahuchet \& Lizet 2001).

18. Cette critique est largement partagée par d'autres ethnologues (Voir Piault 2001, Jolly 2001).

19. Notons qu'elle a scrupuleusement tenu son cahier de terrain jusqu'à son dernier séjour en 1998, alors âgée de 95 ans.

20. A.GD 17, p. 453 à 456 du carnet 1953. M.-N. Bourguet $(2010: 384)$ a également distingué des styles d'écriture différents selon les moments de l'observation dans les journaux des voyageurs des XVIII et XIX ${ }^{\mathrm{e}}$ siècles.

21. Clifford, cité dans Doquet (2009: 53), repère trois moments dans l'écriture des notes de terrain : l'inscription de ce qui vient d'être dit, la transcription mot pour mot des propos de l'informateur, puis la description qui consiste à "produire une représentation relativement cohérente de la réalité culturelle ». L'enquête ethnobotanique comprend un voire deux moments supplémentaires avec la collecte et la liste des plantes qui ensuite permet le dialogue avec l'informateur.

22. A.GD 10 et rapport de mission 1953, A.GD 20. Cette bipartition des échantillons collectés entre deux institutions semble caractériser les collectes des ethnologues et souligne leur ambivalence entre spécimen naturel et objet ethnographique. Voir Bondaz (2018: 359) et Brousse (2017).

23. Lettre du 20/11/1954 de T. Monod à M. Griaule en mission à Sanga (A.GD 10).

24. Lettre du laboratoire de Pharmacologie et de matière médicale, faculté de médecine de Paris, à Madame Dieterlen à Sanga 12 octobre 1953, A.GD 10.

25. Ce travail serait à prolonger par des recherches dans les archives de la faculté de pharmacie.

26. C. Friedberg (1933-2018), première ethnologue à entrer au Muséum dans le laboratoire dirigé par R. Portères a été recrutée en 1956 (Bahuchet 2018).

27. Selon J. Bondaz (2018: 350), A.Chevalier était déjà proche d'ethnologues au sein de l' Association Colonie Sciences créée en 1926, et de la Société des Africanistes. Il a publié plusieurs articles dans le journal de cette Société et M. Griaule a quant à lui publié dans la Revue de Botanique Appliquée, créée par A. Chevalier en 1921. Les archives de ce laboratoire conservent des traces des relations entre ethnologues et botanistes-agronomes: leurs correspondances, la présence conjointe sur les étiquettes des échantillons collectés des noms des ethnologues et des botanistes qui ont fait les déterminations botaniques.

28. Rapport d'activités de 1959, A.GD 20.

29. Albert Atcho s'inscrit dans le mouvement messianique fondé par le prophète William Harris (1865-1929) en 1913. Originaire du Liberia, celui-ci a converti en peu de temps plus de 100000 personnes en Côte d'Ivoire, impulsant une « révolution » culturelle par l'abandon des pratiques religieuses locales. Atcho se désigne lui-même comme prophète, inspiré par Harris tout en s'en distinguant par la création en 1948 d'une institution thérapeutico-religieuse basée sur les « confessions diaboliques » et les soins pharmaceutiques à base de plantes (Rouch 1963).

30. Rapport de mission de 1959, A.GD 20. Dès 1959, G. Dieterlen qualifie le laboratoire de Portères « d'ethno-botanique », ce qu'il ne devient officiellement qu'en 1963.

31. Leurs recherches s'inscrivent dans le programme sur les plantes médicinales décrit par R. Portères (1957).

32. Voir Bouquet et Debray (1974: 143).

33. A. Bouquet et M. Debray sont restés trois ans en Côte d'Ivoire à partir de 1957.Ils ont poursuivi les enquêtes sur les plantes médicinales entreprises par Numa Laffite de 1935 à 1940 et Joseph Kerharo en 1947.

34. Jusqu'à sa mort, G. Dieterlen a entretenu une relation amicale étroite avec le cinéaste et anthropologue Jean Rouch.

35. Rapport de mission 1962 A.GD 20.

36. Sur la genèse et le déroulé de cette enquête, voir Rouch 1963 et 1975. 
37. Rouch 1975 ; rapport de mission 1962 A.GD 20.

38. Une enquête récente menée à Bregbo par une étudiante en master 2 en anthropologie de la santé (CEPED Université Paris Descartes), F. Hellweg (2019), a révélé l'existence d'un «herbier Atcho ", sauvegardé par ses anciens disciples, collaborateurs et ses enfants (Hellweg 2019 : 47). Information recueillie auprès de Basile Atcho, fils d'Albert Atcho installé à Bregbo comme thérapeute spécialisé dans les maladies mentales au Centre Thérapeutique Atcho. L'herbier d'Atcho est à rechercher auprès des anciens collaborateurs et de leurs descendants.

39. Hubert Gillet (1924-2009), agronome de formation et botaniste spécialiste du Sahara, est entré au Muséum comme assistant de R. Portères en 1948 (Boulvert 2011).

40. Un double de ces fiches se trouve dans les archives: A.GD 3. Près de 500 échantillons sont conservés au MNHN dans la collection d'ethnobotanique. Voir Annexe 1.

41. A.GD 20 - Dossier pharmacopée, sous-dossier pharmacopée Bregbo.

42. A.GD 3 : avant-propos de G. Dieterlen ; Jean Rouch (1975:26) dans son avant-propos, présente les apports de l'équipe pluridisciplinaire et reconnaît aussi que l'enquête ethnobotanique, dirigée par G. Dieterlen a été réalisée par Jean Vangah, secrétaire de Atcho.

43. CR Réunion26/06/1967 A.GD 3.

44. Ce qu'elle précise dans les brouillons de son avant-propos et de l'introduction rédigée pour la publication (A.GD 3). L'analyse de ces interprétations relève de l'anthropologie de la santé et constitue une piste de recherche.

45. Son nom identifié à partir de sa signature n'est pas certain.

46. A.GD 20.Roger Heim (1900-1979), ami du pharmacologue Faral, était lui-même ingénieur chimiste de formation de l'École Centrale de Paris. Heim a finalement fait carrière au Muséum dont il a été le Directeur de 1951 à 1965, spécialiste en hallucinogènes (Chadefaud 1979).

47. Voir Crémieux Brilhac1995. H. Laugier a œuvré toute sa vie pour mettre la science au service de la société. Il est membre fondateur de l'Institut d'Études du Développement Économique et Social (IEDES) et de la revue Tiers-monde en 1957. L'éducation et la recherche étaient au cœur de ses préoccupations. Il a d'ailleurs participé au colloque d'Abidjan en 1959 à la suite duquel il a prôné la création d'une« Université internationale au service des pays sous-développés ». Voir aussi Fischer \& Laugier1960, Laugier 1966.

48. A.GD 20. Mots soulignés par Laugier.

49. Les propos de Laugier sont à resituer dans le contexte politique de l'époque. Il n'était pas question alors de pillage des ressources et de biopiraterie mais au contraire d'inventorier les ressources pour le bien de tous. Critique vis-à-vis de «l'Empire » et de la colonisation, il ne peut cependant s'empêcher d'utiliser l'expression alors commune " nos colonies » pour désigner les pays africains indépendants depuis quelques années seulement.

50. Sa correspondance avec des ingénieurs ou des médecins montre son intérêt pour l'application de ses recherches dans les secteurs de la santé et de l'économie. Elle a notamment participé à l'aménagement d'un barrage en pays dogon à Sanga. Elle a rédigé un rapport pour le Ministère de la France d'outre-mer (1953) dans lequel elle précise que ce projet de barrage a été initié par « les missions Griaule» et a été réalisé avec la participation bénévole de la population. Selon G. Dieterlen, les conséquences sociales ont été positives (A.GD 20).

51. Le laboratoire Debat a été créé à Garches (92) en 1920 par le docteur François Debat. Son médicament phare, le Tadenan, était produit à partir du Prunus africana utilisé en urologie. Le laboratoire a été vendu en 1992 au laboratoire Fournier, son principal concurrent français, («Fournier rachète les laboratoires Debat », D. Co. -Les Echos, le 08/02/1993).

52. A.GD 10. Ce tableau se réfère au cahier de terrain de 1977 page431 et suivantes.

53. A.GD 17p. 137, 27/11/1973 : Herbier I et II. E. Jolly, lors d'un entretien en août 2019, émet l'hypothèse que la disparition des premiers informateurs dogon entre 1960 et 1973 peut avoir eu un impact sur les collectes botaniques en obligeant $G$. Dieterlen à compter sur des informateurs ou des collecteurs variés. Il explique ainsi l'absence de publication des deux tomes de suite à 
Renard pâle, ses nouveaux informateurs contredisant les informations précédentes au lieu de les compléter.

54. Herbier Sanga 1977, p. 429. A.GD 9. Selon E. Jolly, Amadigné faisait aussi office "d'infirmier » à Sanga. En 1973, c'est l'un des rares survivants des informateurs privilégiés des missions Griaule (com. Orale).

55. La différence d'écriture observée entre la liste de plantes et leurs usages dans le carnet de terrain de 1953 va dans le sens de cette interprétation. Voir supra note 23.

56. G. Dieterlen a d'ailleurs eu un accident lors d'une enquête au Mali comme l'atteste un certificat médical d'un médecin de l'office du Niger datant du 25 octobre 1952. Elle a été plâtrée et immobilisée pendant 45 jours pour une fracture à une jambe après avoir chuté en terrain accidenté, montrant les risques encourus. A.GD 20.

57. Selon J. Bondaz (2018: 363) les ethnologues ont particulièrement utilisé cette flore ainsi qu'un glossaire des noms vernaculaires en plusieurs langues consulté à l'office du Niger dont Roberty (1907-1971) était le Directeur de la section botanique.

58. Dans le même dossier figure un Reçu, daté de 1977/07/17 à Bamako, qui atteste de l'envoi par G. Dieterlen d'une somme de $4000 \mathrm{f}$ pour l'achat de plantes médicinales, dont le Pénu, à I Guindo, d'après sa signature (A.GD 10).

59. A.GD 10, voir aussi carnet de terrain 1979 A.GD 17, p. 479 : herbier 1977-1978 : liste de noms de plantes en langue vernaculaire et sur la même page, herbier 1978-1979 pour Debat : six plantes rapportées : en $\mathrm{kg}$ avec noms scientifiques.

60. Présenté en Annexe.

61. A.GD 10 : Lettre du 05/07/1977 de J.G. Adam (1909-1980) à Joan Bungener.

62. Anne Luxereau (UMR 7206 EA MNHN-CNRS), lors d'un entretien en février 2019 sur la technique de l'herbier utilisée par des ethnologues, m'a indiqué qu'un informateur pour appuyer son discours sur les propriétés d'une plante peut être amené à la frotter, la couper pour en faire sentir l'odeur ou goûter l'amertume. J'ai moi-même observé au Bénin comment les feuilles d'une plante sont pressées pour en extraire le jus et nettoyer les yeux. Ces manipulations favorisent la transmission du savoir mais entraînent la perte de l'échantillon.

63. Il faut cependant distinguer les herbiers des chercheurs ayant suivi une double formation en botanique et ethnologie comme Claudine Friedberg. Voir S. Bahuchet (2018).

64. Une autre explication peut être avancée : l'évolution de l'ethnologie à la fin des années 1950 avec l'émergence du structuralisme initié par C. Levi-Strauss (voir Jolly $2001: 181$ ).

65. Les herbiers conservés dans les collections d'ethnobotanique du MNHN sont de taille et de qualité très variées. La collection Dieterlen n'est qu'un exemple non représentatif de l'ensemble. Voir Bahuchet et al. (2019).

66. Au Centre Thérapeutique Atcho, Basile Atcho n'utilise qu'une seule plante de l'herbier de son père, Rauwolfia vomitoria. Selon F. Hellweg (2019: 87), il a pour projet de créer un jardin botanique et d'y planter les espèces utilisées par A. Atcho. La question de la transmission du savoir se pose donc et des recherches futures à partir de l'herbier de Dieterlen et de celui d'Atcho permettront peut-être de retracer le processus.

67. Je tiens à remercier Eric Jolly et Marianne Lemaire (IMAf), Catherine Hoare, Anne Luxereau et Flora Pennec (EA), Véronique Duchesne (CEPED) et Simon Juraver (Herbier général MNHN) pour leurs commentaires et remarques constructives sur les collections d'ethnobotanique du Muséum, le contexte des missions ethnographiques, la pratique de l'herbier par les ethnologues.

68. Ces herbiers font partie des "collections miniatures" présentées dans l'Inventaire des collections d'ethnobotanique du laboratoire d'Éco-anthropologie et Ethnobiologie réalisé par Paule Teres et Mounir Zeroual du 1er juillet au 30 septembre 2011. 


\section{RÉSUMÉS}

Germaine Dieterlen, ethnologue reconnue internationalement pour ses études sur les systèmes religieux dogon au Mali, a montré tout au long de sa longue carrière, beaucoup d'intérêt pour les plantes médicinales. Elle a ainsi réalisé des enquêtes ethnobotaniques chez les Dogon et les Bambara au Mali mais aussi dans le sud-est de la Côte d'Ivoire. Cet intérêt l'a amenée à contacter des laboratoires pharmacologiques et même à travailler pour eux en collectant des plantes médicinales à des fins d'analyse. Cet aspect resté méconnu de ses recherches est révélé par ses archives et ses herbiers conservés en partie au Muséum national d'Histoire naturelle de Paris. Cette étude met en évidence le rôle des informateurs locaux investis dans les recherches de l'ethnologue, tout particulièrement dans la collecte des échantillons d'herbier. Ceux-ci, étudiés comme des sources, éclairent d'un nouveau regard l'histoire des sciences humaines.

Germaine Dieterlen, an ethnologist internationally recognized for her studies on the Dogon religious systems in Mali, has shown throughout her long career a great interest in medicinal plants. She has thus done ethnobotanical surveys among the Dogon and Bambara in Mali but also in the south-east of the Ivory Coast. This interest led her to contact pharmacological laboratories and even to work for them by collecting medicinal plants for analysis. This little-known aspect of her research is revealed by her archives and herbariums, some of which are kept at the National Museum of Natural History in Paris. This study shows the role of local informants involved in the ethnologist's research, particularly in the collection of herbarium samples. These, studied as sources, highlight the history of the human sciences in a new light.

\section{INDEX}

Keywords : history of sciences, ethnology, botany, pharmacology, herbarium, medicinal plants, collection, Africa, Mali, Ivory Coast

Mots-clés : histoire des sciences, ethnologie, botanique, pharmacologie, herbier, plantes médicinales, collection, Afrique, Mali, Côte d'Ivoire

\section{AUTEUR}

\section{DOMINIQUE JUHÉ-BEAULATON}

Centre Alexandre Koyré - UMR 8560 EHESS - CNRS - MNHN 